SUPPORTING INFORMATION

for

\title{
Modifying Phosphorus(III) Substituents to Activate Remote Ligand- Centered Reactivity in Triaminoborane Ligands
}

Kyounghoon Lee, ${ }^{\dagger}$ Johnathan D. Culpepper, ${ }^{\dagger}$ Riffat Parveen, ${ }^{\ddagger}$ Dale C. Swenson, ${ }^{\dagger}$ Bess Vlaisavljevich, ${ }^{* *}$ Scott R. Daly ${ }^{* *}$

Email: scott-daly@uiowa.edu, Bess.Vlaisavljevich@usd.edu

${ }^{\dagger}$ The University of Iowa, Department of Chemistry, E331 Chemistry Building, Iowa City, IA 52242, United States

The University of South Dakota, Department of Chemistry, 414 E. Clark Street, Vermillion, SD 57069, United States

\section{TABLE OF CONTENTS}

Experimental

Molecular Structures of 1, 2, 5, 6, and 8

Tabulated Crystallographic Data

NMR Spectra

Supporting Information References pp. S2 - S7

pp. S8 - S9

pp. S10

pp. S11 - S3

pp. S54 


\section{Experimental}

General Considerations. Reactions were carried out under an atmosphere of $\mathrm{N}_{2}$ or Ar using glovebox or standard Schlenk techniques unless stated otherwise. Glassware used for reactions performed under inert conditions was dried in an oven at $150{ }^{\circ} \mathrm{C}$ for at least $1.5 \mathrm{~h}$ and allowed to cool under vacuum before use. Solvents used under anhydrous conditions were dried and deoxygenated using a Pure Process Technologies Solvent Purification System. Reagents were purchased from commercial vendors and used without further purification. $\mathrm{MeOH}$ was refluxed over $\mathrm{Mg}$ and distilled before use. $\mathrm{NEt}_{3}$ was distilled and stored over $\mathrm{KOH}$. 1,8,10,9triazaboradecalin (TBD), ${ }^{1} \mathrm{ClP}(\mathrm{OMe})_{2},{ }^{2}$ and ${ }^{\mathrm{Ph}} \mathrm{TBDPhos}^{1}$ were prepared as previously described.

${ }^{1} \mathrm{H},{ }^{19} \mathrm{~F}$, and ${ }^{31} \mathrm{P}$ NMR data were recorded on a Bruker AVANCE-300 or DPX-300 instrument operating at $300 \mathrm{MHz}$ for ${ }^{1} \mathrm{H}, 282.2 \mathrm{MHz}$ for ${ }^{19} \mathrm{~F}$, and $121.4 \mathrm{MHz}$ for ${ }^{31} \mathrm{P} .{ }^{11} \mathrm{~B}$ and ${ }^{13} \mathrm{C}$ NMR data were acquired on a Bruker AVANCE-400 or DRX-400 instrument operating at 128.3 and 75.5 $\mathrm{MHz}$, respectively. Chemical shifts are reported in $\delta$ units in ppm referenced to residual solvent peaks $\left({ }^{1} \mathrm{H}\right.$ and $\left.{ }^{13} \mathrm{C}\right), 85 \% \mathrm{H}_{3} \mathrm{PO}_{4}\left({ }^{31} \mathrm{P} ; \delta 0.0 \mathrm{ppm}\right), 0.05 \% \mathrm{C}_{6} \mathrm{H}_{5} \mathrm{CF}_{3}$ in $\mathrm{C}_{6} \mathrm{D}_{6}\left({ }^{19} \mathrm{~F} ; \delta-62.9 \mathrm{ppm}\right)$, or $\mathrm{BF}_{3} \cdot \mathrm{Et}_{2} \mathrm{O}\left({ }^{11} \mathrm{~B} ; \delta 0.0 \mathrm{ppm}\right)$. Microanalysis data $(\mathrm{CHN})$ were collected using an EAI CE-440 Elemental Analyzer at the University of Iowa. IR spectra were collected on a Thermo Scientific Nicolet iS5 using $\mathrm{KBr}$ pellets or an attenuated total reflection (ATR) accessory in an $\mathrm{N}_{2}$-filled glovebox. HR-EI mass spectra were recorded on a Waters GCT Premier TOF MS instrument.

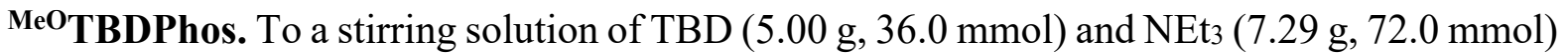
in $\mathrm{CH}_{2} \mathrm{Cl}_{2}(100 \mathrm{~mL})$ was added $\mathrm{ClP}(\mathrm{OMe})_{2}(9.25 \mathrm{~g}, 72.0 \mathrm{mmol})$. The reaction was stirred overnight, evaporated to dryness under vacuum, and extracted with THF (300 mL). The filtrate was evaporated to dryness under vacuum and dissolved in pentane $(40 \mathrm{~mL})$. Cooling the solution to $-30{ }^{\circ} \mathrm{C}$ yielded a white precipitate. Yield: $9.05 \mathrm{~g}(78 \%)$. Anal. Calcd for $\mathrm{C}_{10} \mathrm{H}_{24} \mathrm{BN}_{3} \mathrm{O}_{4} \mathrm{P}_{2}$ : $\mathrm{C}$, 37.2; H, 7.49; N, 13.0. Found: C, 37.0; H, 7.33; N, 13.0. ${ }^{1} \mathrm{H} \mathrm{NMR}\left(\mathrm{CDCl}_{3}, 20{ }^{\circ} \mathrm{C}\right): \delta 1.77$ (quint, $\left.\mathrm{CH}_{2}-\underline{\mathrm{C}}_{2}-\mathrm{CH}_{2}, 4 \mathrm{H}\right), 2.86\left(\mathrm{t}, \mathrm{NCH}_{2}, 4 \mathrm{H}\right), 3.15\left(\mathrm{~m}, \mathrm{NCH}_{2}, 4 \mathrm{H}\right), 3.50\left(\mathrm{t}, \mathrm{OCH}_{3}, 12 \mathrm{H}\right) .{ }^{11} \mathrm{~B} \mathrm{NMR}$ $\left(\mathrm{CDCl}_{3}, 20{ }^{\circ} \mathrm{C}\right): \delta 24.7($ br s, FWHM $=220 \mathrm{~Hz}) .{ }^{13} \mathrm{C}\left\{{ }^{1} \mathrm{H}\right\} \mathrm{NMR}\left(\mathrm{CDCl}_{3}, 20{ }^{\circ} \mathrm{C}\right): \delta 27.9\left(\mathrm{~s}, \mathrm{CH}_{2}-\right.$ $\left.\mathrm{CH}_{2}-\mathrm{CH}_{2}\right), 36.9\left(\mathrm{~s}, \mathrm{NCH}_{2}\right), 48.3\left(\mathrm{~s}, \mathrm{NCH}_{2}\right), 51.9$ (t, $\left.J=11 \mathrm{~Hz}, \mathrm{OCH}_{3}\right) .{ }^{31} \mathrm{P}\left\{{ }^{1} \mathrm{H}\right\} \mathrm{NMR}\left(\mathrm{CDCl}_{3}, 20\right.$ ${ }^{\circ} \mathrm{C}$ ): $\delta 145.6(\mathrm{~s})$. MS (EI) $\mathrm{m} / \mathrm{z}$ : calcd for $\mathrm{C}_{10} \mathrm{H}_{24} \mathrm{BN}_{3} \mathrm{O}_{4} \mathrm{P}_{2} 323.1335$; found 323.1351. IR (ATR, $\mathrm{cm}^{-}$ 1): $2978 \mathrm{w}, 2951 \mathrm{w}, 2925 \mathrm{~m}, 2861 \mathrm{w}, 2822 \mathrm{~m}, 1527 \mathrm{w}, 1513 \mathrm{~m}, 1470 \mathrm{~m}, 1442 \mathrm{~m}, 1371 \mathrm{~s}, 1360 \mathrm{w}$, 1349 w, 1322 m, 1310 w, 1295 s, 1229 w, 1209 m, 1166 m, 1113 m, 1098 m, 1019 vs, 972 m, 917 vw, 903 w, 879 m, 846 vw, 814 m, 798 vw, 764 w, 754 w, 745 w, 738 w, 695 vs, 684 vw, 671 vw, $661 \mathrm{~m}, 651 \mathrm{~m}$.

EtTBDPhos. To a stirring solution of TBD $(0.20 \mathrm{~g}, 1.4 \mathrm{mmol})$ and NEt $3(0.29 \mathrm{~g}, 2.9 \mathrm{mmol})$ in $\mathrm{CH}_{2} \mathrm{Cl}_{2}(30 \mathrm{~mL})$ was added $\mathrm{ClPEt}_{2}(0.36 \mathrm{~g}, 2.9 \mathrm{mmol})$. The reaction was stirred overnight, evaporated to dryness under vacuum, and extracted with THF $(40 \mathrm{~mL})$. The filtrate was evaporated to dryness under vacuum and dissolved in pentane $(5 \mathrm{~mL})$. The solution was cooled to $-30{ }^{\circ} \mathrm{C}$ to yield colorless needles. Yield: $0.18 \mathrm{~g}(40 \%)$. ${ }^{1} \mathrm{H} \mathrm{NMR}\left(\mathrm{CDCl}_{3}, 20{ }^{\circ} \mathrm{C}\right): \delta 1.01\left(\mathrm{~m},-\mathrm{CH}_{2}-\mathrm{C}_{3}\right.$, $12 \mathrm{H}), 1.34\left(\mathrm{~m},-\mathrm{C}_{2}-\mathrm{CH}_{3}, 4 \mathrm{H}\right), 1.64\left(\mathrm{~m},-\mathrm{CH}_{2}-\mathrm{CH}_{3}, 4 \mathrm{H}\right), 1.70\left(\mathrm{~m}, \mathrm{CH}_{2}-\mathrm{C}_{2}-\mathrm{CH}_{2}, 4 \mathrm{H}\right), 2.83(\mathrm{t}$, $\left.\mathrm{NCH}_{2}, 4 \mathrm{H}\right), 2.93\left(\mathrm{~m}, \mathrm{NCH}_{2}, 4 \mathrm{H}\right) .{ }^{11} \mathrm{~B} \mathrm{NMR}\left(\mathrm{CDCl}_{3}, 20{ }^{\circ} \mathrm{C}\right): \delta 25.9$ (br s, FWHM = $\left.220 \mathrm{~Hz}\right)$. ${ }^{13} \mathrm{C}\left\{{ }^{1} \mathrm{H}\right\}$ NMR $\left(\mathrm{CDCl}_{3}, 20{ }^{\circ} \mathrm{C}\right): \delta 9.9\left(\mathrm{~s},-\mathrm{CH}_{2}-\mathrm{CH}_{3}\right), 20.5\left(\mathrm{~s},-\mathrm{CH}_{2}-\mathrm{CH}_{3}\right), 28.4\left(\mathrm{~s}, \mathrm{CH}_{2}-\mathrm{CH}_{2}-\right.$ $\left.\mathrm{CH}_{2}\right), 40.1$ (s, $\left.\mathrm{NCH}_{2}\right), 48.7\left(\mathrm{~s}, \mathrm{NCH}_{2}\right) .{ }^{31} \mathrm{P}\left\{{ }^{1} \mathrm{H}\right\} \mathrm{NMR}\left(\mathrm{CDCl}_{3}, 20{ }^{\circ} \mathrm{C}\right): \delta 43.3(\mathrm{~s})$.

( $\left.{ }^{\mathrm{Ph}} \mathbf{T B D P h o s}\right) \mathrm{PtCl}_{2}$ (1). To a stirring solution of (COD) $\mathrm{PtCl}_{2}(0.68 \mathrm{~g}, 1.3 \mathrm{mmol})$ in $\mathrm{CH}_{2} \mathrm{Cl}_{2}(50$ $\mathrm{mL})$ was added a solution of ${ }^{\mathrm{Ph}}$ TBDPhos $(0.50 \mathrm{~g}, 1.3 \mathrm{mmol})$ in $\mathrm{CH}_{2} \mathrm{Cl}_{2}(50 \mathrm{~mL})$. The reaction was stirred overnight, and hexane was added to induce formation of a white precipitate. The precipitate 
was isolated by filtration, dissolved in $\mathrm{CH}_{2} \mathrm{Cl}_{2}$, and crystallized by vapor diffusion with $\mathrm{Et}_{2} \mathrm{O}$. Yield: $0.92 \mathrm{~g}(89 \%)$. Anal. Calcd for $\mathrm{C}_{30} \mathrm{H}_{32} \mathrm{BCl}_{2} \mathrm{~N}_{3} \mathrm{P}_{2} \mathrm{Pt} \cdot 2 \mathrm{CH}_{2} \mathrm{Cl}_{2}$ : C, 40.8; H, 3.85; N, 4.46. Found: C, 40.8; $\mathrm{H}, 3.66 ; \mathrm{N}, 4.60$. The two molecules of $\mathrm{CH}_{2} \mathrm{Cl}_{2}$ included in the EA are present in the XRD structure and observed in the ${ }^{1} \mathrm{H}$ NMR spectrum. ${ }^{1} \mathrm{H}$ NMR $\left(\mathrm{CDCl}_{3}, 20{ }^{\circ} \mathrm{C}\right): \delta 1.54(\mathrm{~m}$, $\left.\mathrm{CH}_{2}-\mathrm{CH}_{2}-\mathrm{CH}_{2}, 4 \mathrm{H}\right), 2.81-2.93\left(\mathrm{~m}, \mathrm{NCH}_{2}, 8 \mathrm{H}\right), 7.33-7.47(\mathrm{~m}, \mathrm{Ph}, 12 \mathrm{H}), 7.70-7.81(\mathrm{~m}, \mathrm{Ph}$, $8 \mathrm{H}) .{ }^{11} \mathrm{~B} \mathrm{NMR}\left(\mathrm{CDCl}_{3}, 20{ }^{\circ} \mathrm{C}\right): \delta 24.4$ (br s, FWHM $\left.=460 \mathrm{~Hz}\right) \cdot{ }^{13} \mathrm{C}\left\{{ }^{1} \mathrm{H}\right\} \mathrm{NMR}\left(\mathrm{CDCl}_{3}, 20{ }^{\circ} \mathrm{C}\right): \delta$ $26.0\left(\mathrm{~s}, \mathrm{CH}_{2}-\mathrm{CH}_{2}-\mathrm{CH}_{2}\right), 47.0\left(\mathrm{~s}, \mathrm{NCH}_{2}\right), 48.3\left(\mathrm{~s}, \mathrm{NCH}_{2}\right), 128.3$ (t, J = 6.0 Hz, Ph), $131.1(\mathrm{~s}, \mathrm{Ph})$, $133.1(\mathrm{t}, J=5.3 \mathrm{~Hz}, \mathrm{Ph}) .{ }^{31} \mathrm{P}\left\{{ }^{1} \mathrm{H}\right\} \mathrm{NMR}\left(\mathrm{CDCl}_{3}, 20{ }^{\circ} \mathrm{C}\right): \delta 43.9$ (s with doublet satellites, ${ }^{1} J_{\mathrm{PtP}}=$ $3730 \mathrm{~Hz}$ ). MS (EI) $\mathrm{m} / \mathrm{z}$ : calcd for $\mathrm{C}_{30} \mathrm{H}_{32} \mathrm{BCl}_{2} \mathrm{~N}_{3} \mathrm{P}_{2} \mathrm{Pt} 772.1189$; found 772.1248. IR (ATR, $\mathrm{cm}^{-1}$ ): 3050 w, 2979 vw, 2945 w, 2859 vw, 1588 vw, 1536 m, 1519 s, 1481 w, 1466 w, 1448 vw, 1432 s, $1384 \mathrm{~m}, 1363 \mathrm{~m}, 1348 \mathrm{w}, 1324 \mathrm{~m}, 1298 \mathrm{~m}, 1280 \mathrm{~m}, 1268 \mathrm{w}, 1258 \mathrm{w}, 1210 \mathrm{~s}, 1172 \mathrm{~m}, 1119 \mathrm{vw}$, 1097 s, 1058 w, 1027 s, 1011 vw, 997 w, 917 m, 892 m, 833 m, 788 m, 743 s, 722 s, 715 vw, 690 vs, $639 \mathrm{~m}, 629 \mathrm{w}, 620 \mathrm{~m}, 605 \mathrm{~s}, 581 \mathrm{~s}, 576 \mathrm{~m}, 559 \mathrm{w}, 555 \mathrm{~s}$.

( ${ }^{\mathrm{MeO}} \mathrm{TBDPhos}_{\mathbf{P}} \mathrm{PtCl}_{2}$ (2). To a stirring solution of (COD)PtCl $2(0.38 \mathrm{~g}, 1.0 \mathrm{mmol})$ in $\mathrm{CH}_{2} \mathrm{Cl}_{2}$ $(5 \mathrm{~mL})$ was added a solution of ${ }^{\mathrm{MeO}} \mathrm{TBDPhos}(0.33 \mathrm{~g}, 1.0 \mathrm{mmol})$ in $\mathrm{CH}_{2} \mathrm{Cl}_{2}(5 \mathrm{~mL})$. The reaction was stirred overnight, reduced to $5 \mathrm{~mL}$, and crystallized by vapor diffusion with $\mathrm{Et}_{2} \mathrm{O}$ to yield colorless needles. Yield: $0.57 \mathrm{~g}(95 \%)$. Anal. Calcd for $\mathrm{C}_{10} \mathrm{H}_{24} \mathrm{BCl}_{2} \mathrm{~N}_{3} \mathrm{O}_{4} \mathrm{P}_{2} \mathrm{Pt}$ : C, 20.4; $\mathrm{H}, 4.11 ; \mathrm{N}$, 7.13. Found: $\mathrm{C}, 20.5 ; \mathrm{H}, 3.93 ; \mathrm{N}, 6.84 .{ }^{1} \mathrm{H} \mathrm{NMR}\left(\mathrm{CDCl}_{3}, 20{ }^{\circ} \mathrm{C}\right.$ ): $\delta 1.85$ (quint, $\mathrm{CH}_{2}-\mathrm{CH}_{2}-\mathrm{CH}_{2}$, $4 \mathrm{H}), 2.94\left(\mathrm{t}, \mathrm{NCH}_{2}, 4 \mathrm{H}\right), 3.23\left(\mathrm{~m}, \mathrm{NCH}_{2}, 4 \mathrm{H}\right), 3.86\left(\mathrm{t}, \mathrm{OCH}_{3}, 12 \mathrm{H}\right) .{ }^{11} \mathrm{~B} \mathrm{NMR}\left(\mathrm{CDCl}_{3}, 20{ }^{\circ} \mathrm{C}\right): \delta$ $22.7($ br s, FWHM $=260 \mathrm{~Hz}) \cdot{ }^{13} \mathrm{C}\left\{{ }^{1} \mathrm{H}\right\} \mathrm{NMR}\left(\mathrm{CDCl}_{3}, 20^{\circ} \mathrm{C}\right): \delta 26.0\left(\mathrm{~s}, \mathrm{CH}_{2}-\mathrm{CH}_{2}-\mathrm{CH}_{2}\right), 40.5(\mathrm{~s}$, $\left.\mathrm{NCH}_{2}\right), 48.6\left(\mathrm{~s}, \mathrm{NCH}_{2}\right), 54.6\left(\mathrm{~s}, \mathrm{OCH}_{3}\right) .{ }^{31} \mathrm{P}\left\{{ }^{1} \mathrm{H}\right\} \mathrm{NMR}\left(\mathrm{CDCl}_{3}, 20{ }^{\circ} \mathrm{C}\right): \delta 69.9$ (s with doublet satellites, ${ }^{1} J_{\mathrm{PtP}}=4895 \mathrm{~Hz}$ ). MS (EI) $\mathrm{m} / \mathrm{z}$ : calcd for $\mathrm{C}_{10} \mathrm{H}_{24} \mathrm{BCl}_{2} \mathrm{~N}_{3} \mathrm{O}_{4} \mathrm{P} 2 \mathrm{Pt}$ 588.0360; found 588.0389. IR (ATR, cm ${ }^{-1}$ ): $3003 \mathrm{w}, 2904 \mathrm{w}, 2880 \mathrm{w}, 2853 \mathrm{w}, 1524 \mathrm{vw}, 1509 \mathrm{~m}, 1466 \mathrm{vw}, 1454$ w, $1445 \mathrm{vw}, 1428 \mathrm{vw}, 1390 \mathrm{~m}, 1364 \mathrm{~m}, 1351 \mathrm{w}, 1322 \mathrm{~m}, 1306 \mathrm{w}, 1293 \mathrm{vw}, 1285 \mathrm{~m}, 1207 \mathrm{~m}, 1169$ m, $1113 \mathrm{vw}, 1088$ m, 1064 vw, 1054 vw, 1024 vw, 1004 vs, 922 s, 890 w, 875 vw, 864 vw, 856 vw, $837 \mathrm{w}, 811 \mathrm{~s}, 775 \mathrm{~s}, 749 \mathrm{vw}, 741 \mathrm{vw}, 730 \mathrm{w}, 722 \mathrm{w}, 713 \mathrm{vw}, 703 \mathrm{vw}, 693 \mathrm{vw}, 698 \mathrm{vw}, 683$ w, 673 w, 665 w, 655 m, $646 \mathrm{~m}, 637 \mathrm{~s}, 630 \mathrm{~s}, 621 \mathrm{~s}, 612 \mathrm{~s}$.

( ${ }^{\text {Et }} \mathbf{T B D P h o s} \mathbf{P t C l}_{2}$ (3). To a stirring solution of ${ }^{\mathrm{Et}} \mathrm{TBDPhos}(0.14 \mathrm{~g}, 0.44 \mathrm{mmol})$ in $\mathrm{CH}_{2} \mathrm{Cl}_{2}(7$ $\mathrm{mL})$ was added (COD) $\mathrm{PtCl}_{2}(0.17 \mathrm{~g}, 0.45 \mathrm{mmol})$. The reaction was stirred overnight, reduced to $\sim 4 \mathrm{~mL}$, and crystallized by vapor diffusion with $\mathrm{Et}_{2} \mathrm{O}$ to yield colorless needles. Yield: $0.18 \mathrm{~g}$ (71\%). ${ }^{1} \mathrm{H} \mathrm{NMR}\left(\mathrm{CDCl}_{3}, 20{ }^{\circ} \mathrm{C}\right): \delta 1.19\left(\mathrm{~m},-\mathrm{CH}_{2}-\mathrm{C}_{3}, 12 \mathrm{H}\right), 1.83\left(\mathrm{~m},-\mathrm{C}_{2}-\mathrm{CH}_{3}, 4 \mathrm{H}\right), 2.00(\mathrm{~m}$, $\left.-\mathrm{CH}_{2}-\mathrm{CH}_{3}, 4 \mathrm{H}\right), 2.61\left(\mathrm{~m}, \mathrm{CH}_{2}-\mathrm{CH}_{2}-\mathrm{CH}_{2}, 4 \mathrm{H}\right), 2.93\left(\mathrm{t}, \mathrm{NCH}_{2}, 4 \mathrm{H}\right), 3.10\left(\mathrm{~m}, \mathrm{NCH}_{2}, 4 \mathrm{H}\right) .{ }^{11} \mathrm{~B}$ $\mathrm{NMR}\left(\mathrm{CDCl}_{3}, 20{ }^{\circ} \mathrm{C}\right): \delta 24.1($ br s, FWHM $=292 \mathrm{~Hz}) \cdot{ }^{13} \mathrm{C}\left\{{ }^{1} \mathrm{H}\right\} \mathrm{NMR}\left(\mathrm{CDCl}_{3}, 20{ }^{\circ} \mathrm{C}\right): \delta 8.9(-$ $\left.\mathrm{CH}_{2}-\underline{\mathrm{CH}}_{3}\right), 20.5\left(\mathrm{~m},-\underline{\mathrm{CH}}_{2}-\mathrm{CH}_{3}\right), 26.7$ (s, $\left.\mathrm{CH}_{2}-\mathrm{CH}_{2}-\mathrm{CH}_{2}\right), 42.5\left(\mathrm{~s}, \mathrm{NCH}_{2}\right), 48.8\left(\mathrm{~s}, \mathrm{NCH}_{2}\right)$. ${ }^{31} \mathrm{P}\left\{{ }^{1} \mathrm{H}\right\} \mathrm{NMR}\left(\mathrm{CDCl}_{3}, 20{ }^{\circ} \mathrm{C}\right): \delta 58.9$ (s with doublet satellites, $\left.{ }^{1} J_{\mathrm{PtP}}=3692 \mathrm{~Hz}\right)$.

( $\left.{ }^{\mathbf{P h}} \mathbf{T B D P h o s}\right) \mathbf{P t}\left(\mathbf{S}_{2} \mathbf{C}_{6} \mathbf{H}_{4}\right)$ (4). To a stirring solution of $\mathbf{1}(0.10 \mathrm{~g}, 0.13 \mathrm{mmol})$ and excess $\mathrm{NEt}_{3}$ $(0.5 \mathrm{~mL})$ in $\mathrm{CH}_{2} \mathrm{Cl}_{2}(5 \mathrm{~mL})$ was added a solution of 1,2-benzenedithiol $(0.018 \mathrm{~g}, 0.13 \mathrm{mmol})$ in $\mathrm{CH}_{2} \mathrm{Cl}_{2}(5 \mathrm{~mL})$. The reaction was stirred overnight, evaporated to dryness under vacuum, and extracted with THF $(10 \mathrm{~mL})$. The solution was evaporated to dryness under vacuum and dissolved in $\mathrm{CH}_{2} \mathrm{Cl}_{2}(5 \mathrm{~mL})$. Vapor diffusion with $\mathrm{Et}_{2} \mathrm{O}$ yielded green-yellow blocks. Yield: $0.080 \mathrm{~g}(73 \%)$. Anal. Calcd for $\mathrm{C}_{36} \mathrm{H}_{36} \mathrm{BN}_{3} \mathrm{P}_{2} \mathrm{PtS}_{2}$ : C, 51.3; H, 4.31; N, 4.99. Found: C, 51.2; H, 4.22; N, 4.57. ${ }^{1} \mathrm{H}$ $\operatorname{NMR}\left(\mathrm{CDCl}_{3}, 2{ }^{\circ} \mathrm{C}\right): \delta 1.62$ (quint, $\left.\mathrm{CH}_{2}-\mathrm{CH}_{2}-\mathrm{CH}_{2}, 4 \mathrm{H}\right), 2.84-3.02\left(\mathrm{~m}, \mathrm{NCH}_{2}, 8 \mathrm{H}\right), 6.57-6.69$ (m, Ar, 2H), $7.18-7.37$ (m, Ar, 14H), $7.48-7.65$ (m, Ar, 8H). ${ }^{11} \mathrm{~B} \mathrm{NMR}\left(\mathrm{CDCl}_{3}, 20{ }^{\circ} \mathrm{C}\right): \delta 24.9$ (br s, FWHM $=510 \mathrm{~Hz}) .{ }^{13} \mathrm{C}\left\{{ }^{1} \mathrm{H}\right\} \mathrm{NMR}\left(\mathrm{CDCl}_{3}, 20{ }^{\circ} \mathrm{C}\right): \delta 26.4\left(\mathrm{~s}, \mathrm{CH}_{2}-\mathrm{CH}_{2}-\mathrm{CH}_{2}\right), 47.2\left(\mathrm{~s}, \mathrm{NCH}_{2}\right)$, 48.6 (s, NCH ), 121.0 (s, Ar), 127.7 (t, J=5.9 Hz, Ar), 129.3 (s, Ar), 130.6 (s, Ar), 131.4 (s, Ar), 
132.1 (s, Ar), 133.1 (t, $J=6.0 \mathrm{~Hz}, \mathrm{Ar}), 146.4(\mathrm{~m}, \mathrm{Ar}) .{ }^{31} \mathrm{P}\left\{{ }^{1} \mathrm{H}\right\} \mathrm{NMR}\left(\mathrm{CDCl}_{3}, 20{ }^{\circ} \mathrm{C}\right): \delta 53.3(\mathrm{~s}$ with doublet satellites, ${ }^{1} J_{\mathrm{PtP}}=2917 \mathrm{~Hz}$ ). $\mathrm{MS}(\mathrm{EI}) \mathrm{m} / \mathrm{z}$ : calcd for $\mathrm{C}_{36} \mathrm{H}_{36} \mathrm{BN}_{3} \mathrm{P}_{2} \mathrm{PtS}_{2}$ 842.1567; found 842.1433. IR (ATR, cm ${ }^{-1}$ ): 3049 vw, 2949 vw, 1559 vw, 1548 vw, 1514 w, $1506 \mathrm{~m}, 1466 \mathrm{w}, 1481$ w, 1443 w, 1432 m, 1417 w, 1383 m, 1360 w, 1348 w, 1323 m, 1298 w, 1266 m, 1236 vw, 1225 vw, $1207 \mathrm{~m}, 1171 \mathrm{~m}, 1095 \mathrm{~s}, 1029 \mathrm{~m}, 997 \mathrm{w}, 937 \mathrm{w}, 905 \mathrm{w}, 891 \mathrm{w}, 830 \mathrm{~m}, 779 \mathrm{~m}, 741 \mathrm{w}, 729 \mathrm{~s}$, $710 \mathrm{~m}, 687 \mathrm{vs}, 663 \mathrm{w}, 656 \mathrm{w}, 635 \mathrm{~m}, 625 \mathrm{vw}, 618 \mathrm{w}, 612 \mathrm{vw}, 601 \mathrm{vw}, 596 \mathrm{~m}, 581 \mathrm{vw}, 569 \mathrm{~m}, 556$ vw, 551 vw. UV-vis $\left(\mathrm{CH}_{2} \mathrm{Cl}_{2}\right) \lambda_{\max }, \mathrm{nm}(\varepsilon): 250\left(5.16 \times 10^{4}\right), 326\left(7.80 \times 10^{3}\right)$.

( ${ }^{\mathrm{MeO}}$ TBDPhos)Pt $\left(\mathbf{S}_{2} \mathbf{C}_{6} \mathbf{H}_{4}\right)$ (5). To a stirring solution of $\mathbf{2}(1.00 \mathrm{~g}, 1.70 \mathrm{mmol})$ and excess $\mathrm{NEt}_{3}$ $(5 \mathrm{~mL})$ in $\mathrm{CH}_{2} \mathrm{Cl}_{2}(30 \mathrm{~mL})$ was added a solution of 1,2-benzenedithiol $(0.24 \mathrm{~g}, 1.7 \mathrm{mmol})$ in $\mathrm{CH}_{2} \mathrm{Cl}_{2}(10 \mathrm{~mL})$. The reaction was stirred overnight, evaporated to dryness under vacuum, and extracted with THF (100 mL). The solution was evaporated to dryness under vacuum and dissolved in $\mathrm{CH}_{2} \mathrm{Cl}_{2}(10 \mathrm{~mL})$. A pale-yellow precipitate was obtained by adding excess $\mathrm{Et}_{2} \mathrm{O}$ to the solution. The precipitate was dissolved in $\mathrm{CH}_{2} \mathrm{Cl}_{2}$ and crystals were grown by vapor diffusion with $\mathrm{Et}_{2} \mathrm{O}$. Yield: $0.82 \mathrm{~g}$ (73\%). Anal. Calcd for $\mathrm{C}_{16} \mathrm{H}_{28} \mathrm{BN}_{3} \mathrm{O}_{4} \mathrm{P}_{2} \mathrm{PtS}_{2}$ : C, 29.2; H, 4.29; N, 6.38. Found: C, 29.3; H, 4.23; N, 6.38. ${ }^{1} \mathrm{H}$ NMR $\left(\mathrm{CDCl}_{3}, 20{ }^{\circ} \mathrm{C}\right): \delta 1.89\left(\mathrm{~m}, \mathrm{CH}_{2}-\mathrm{CH}_{2}-\mathrm{CH}_{2}, 4 \mathrm{H}\right), 2.97\left(\mathrm{t}, \mathrm{NCH}_{2}\right.$, $4 \mathrm{H}), 3.28\left(\mathrm{~m}, \mathrm{NCH}_{2}, 4 \mathrm{H}\right), 3.72-3.84\left(\mathrm{~m}, \mathrm{OCH}_{3} 12 \mathrm{H}\right), 6.78-6.89(\mathrm{~m}, \mathrm{Ar}, 2 \mathrm{H}), 7.57-7.67(\mathrm{~m}$, Ar, 2H). ${ }^{11} \mathrm{~B}$ NMR $\left(\mathrm{CDCl}_{3}, 20{ }^{\circ} \mathrm{C}\right): \delta 23.2$ (br s, FWHM = 410 Hz). ${ }^{13} \mathrm{C}\left\{{ }^{1} \mathrm{H}\right\} \mathrm{NMR}\left(\mathrm{CDCl}_{3}, 20\right.$ $\left.{ }^{\circ} \mathrm{C}\right): \delta 26.1\left(\mathrm{~s}, \mathrm{CH}_{2}-\mathrm{CH}_{2}-\mathrm{CH}_{2}\right), 39.5\left(\mathrm{~s}, \mathrm{NCH}_{2}\right), 48.9\left(\mathrm{~s}, \mathrm{NCH}_{2}\right), 53.9\left(\mathrm{~m}, \mathrm{OCH}_{3}\right), 121.9(\mathrm{~s}, \mathrm{Ar})$, 129.8 (s, Ar), 144.8 (m, Ar). ${ }^{31} \mathrm{P}\left\{{ }^{1} \mathrm{H}\right\}$ NMR $\left(\mathrm{CDCl}_{3}, 20{ }^{\circ} \mathrm{C}\right): \delta 97.7$ (s with doublet satellites, ${ }^{1} J_{\mathrm{PtP}}$ $=3918 \mathrm{~Hz}$ ). MS (EI) $\mathrm{m} / \mathrm{z}$ : calcd for $\mathrm{C}_{16} \mathrm{H}_{28} \mathrm{BN}_{3} \mathrm{O}_{4} \mathrm{P}_{2} \mathrm{PtS}_{2}$ 658.0737; found 658.0807. IR (ATR, $\mathrm{cm}^{-}$ 1): 3016 w, 2937 w, 2872 w, 2834 w, 1535 vw, 1521 m, 1474 w, 1444 m, 1417 w, 1386 m, 1368 m, 1325 m, 1299 w, 1280 s, 1239 vw, 1232 vw, 1210 m, 1176 m, 1121 w, 1096 m, 1065 w, 1029 s, $1000 \mathrm{vs,} 949$ vw, 938 w, 921 m, 899 vw, 891 vw, 834 m, 805 w, 789 s, 760 s, 743 s, 699 vw, $665 \mathrm{w}, 654 \mathrm{vw}$. UV-vis $\left(\mathrm{CH}_{2} \mathrm{Cl}_{2}\right) \lambda_{\max }, \mathrm{nm}(\varepsilon): 249\left(4.58 \times 10^{4}\right), 320\left(1.10 \times 10^{4}\right)$.

( ${ }^{\mathrm{MeO}}$ TBDPhos-MeOH)Pt $\left(\mathbf{S}_{2} \mathbf{C}_{6} \mathbf{H}_{4}\right)$ (5-MeOH). To a stirring solution of $\mathbf{5}(0.10 \mathrm{~g}, 0.15 \mathrm{mmol})$ in $\mathrm{CH}_{2} \mathrm{Cl}_{2}(5 \mathrm{~mL})$ was added $\mathrm{MeOH}(1 \mathrm{~mL})$ and $\mathrm{NEt}_{3}(0.5 \mathrm{~mL})$ in air. The reaction was shaken several times, filtered, and crystallized by vapor diffusion with $\mathrm{Et}_{2} \mathrm{O}$ to yield colorless blocks and needles. Yield: $0.089 \mathrm{~g}(85 \%)$. Anal. Calcd for $\mathrm{C}_{17} \mathrm{H}_{32} \mathrm{BN}_{3} \mathrm{O}_{5} \mathrm{P}_{2} \mathrm{PtS}_{2}$ : C, 29.6; H, 4.67; N, 6.09. Found: $\mathrm{C}, 29.5 ; \mathrm{H}, 4.43 ; \mathrm{N}, 5.51 .{ }^{1} \mathrm{H} \mathrm{NMR}\left(\mathrm{CDCl}_{3}, 20{ }^{\circ} \mathrm{C}\right): \delta 1.66-1.94(\mathrm{~m}, 4 \mathrm{H}), 2.80-3.05(\mathrm{~m}$, $4 \mathrm{H}), 3.06-3.16(\mathrm{~m}, 2 \mathrm{H}), 3.18\left(\mathrm{~s}, \mathrm{~B}-\mathrm{OCH}_{3}, 3 \mathrm{H}\right), 3.59-3.71(\mathrm{~m}, 2 \mathrm{H}), 3.71-3.83\left(\mathrm{~m}, \mathrm{P}\left(\mathrm{OCH}_{3}\right)_{2}\right.$, 12H), 6.78 (m, Ar, 2H), $7.03(\mathrm{~m}, \mathrm{NH}, 1 \mathrm{H}), 7.61(\mathrm{~m}, \mathrm{Ar}, 2 \mathrm{H}) .{ }^{11} \mathrm{~B}$ NMR $\left(\mathrm{CDCl}_{3}, 20{ }^{\circ} \mathrm{C}\right): \delta 1.7(\mathrm{~s})$. ${ }^{13} \mathrm{C}\left\{{ }^{1} \mathrm{H}\right\}$ NMR $\left(\mathrm{CDCl}_{3}, 20{ }^{\circ} \mathrm{C}\right): \delta 27.8(\mathrm{~s}), 38.3(\mathrm{~s}), 47.0(\mathrm{~s}), 50.5(\mathrm{~s}), 54.2(\mathrm{~s}), 121.5(\mathrm{~s}, \mathrm{Ar}), 129.8$ (s, Ar), 145.4 (s, Ar). ${ }^{31} \mathrm{P}\left\{{ }^{1} \mathrm{H}\right\} \mathrm{NMR}\left(\mathrm{CDCl}_{3}, 20{ }^{\circ} \mathrm{C}\right): \delta 97.3$ (s with doublet satellites, ${ }^{1} J_{\mathrm{PtP}}=3954$ Hz). IR (ATR, cm ${ }^{-1}$ ): 2929 w, $2880 \mathrm{w}, 2823 \mathrm{~m}, 1546 \mathrm{w}, 1535 \mathrm{vw}, 1529 \mathrm{vw}, 1512 \mathrm{w}, 1501 \mathrm{vw}$, $1493 \mathrm{vw}, 1484 \mathrm{vw}, 1460 \mathrm{w}, 1444 \mathrm{~m}, 1396 \mathrm{w}, 1359 \mathrm{~m}, 1343 \mathrm{w}, 1293 \mathrm{~m}, 1243 \mathrm{~m}, 1184 \mathrm{~m}, 1138 \mathrm{~s}$, 1103 s, 1079 m, 1041 w, 1019 vs, 973 vw, 909 m, 879 s, 834 m, 802 s, 766 vw, 743 vw, 672 vw, $663 \mathrm{~m}, 636 \mathrm{vw}, 609 \mathrm{w}, 600 \mathrm{w}, 584 \mathrm{w}, 571 \mathrm{~m}, 554 \mathrm{~s}$.

( $\left.{ }^{\text {Et }} \mathbf{T B D P h o s}\right) \mathbf{P t}\left(\mathbf{S}_{\mathbf{2}} \mathbf{C}_{\mathbf{6}} \mathbf{H}_{4}\right)$ (6). To a stirring solution of ( ${ }^{\mathrm{Et}} \mathrm{TBDPhos} \mathrm{PtCl}_{2}(0.15 \mathrm{~g}, 0.26 \mathrm{mmol})$ and $\mathrm{NEt}_{3}(72 \mu \mathrm{L}, 0.52 \mathrm{mmol})$ in $\mathrm{CH}_{2} \mathrm{Cl}_{2}(15 \mathrm{~mL})$ was added a solution of 1,2-benzenedithiol $(0.037$ $\mathrm{g}, 0.26 \mathrm{mmol})$ in $\mathrm{CH}_{2} \mathrm{Cl}_{2}(15 \mathrm{~mL})$. The reaction was stirred overnight, evaporated to dryness under vacuum, and extracted with THF $(20 \mathrm{~mL})$. The solution was then evaporated to dryness under vacuum and dissolved in $\mathrm{CH}_{2} \mathrm{Cl}_{2}(5 \mathrm{~mL})$. Vapor diffusion with $\mathrm{Et}_{2} \mathrm{O}$ yielded greenish-yellow blocks. Yield: $0.11 \mathrm{~g}(66 \%)$. In some reactions it was found that 3 co-crystallizes with 6 if present. ${ }^{1} \mathrm{H}$ NMR $\left(\mathrm{CDCl}_{3}, 20{ }^{\circ} \mathrm{C}\right): \delta 1.20\left(\mathrm{~m},-\mathrm{CH}_{2}-\mathrm{C}_{3}, 12 \mathrm{H}\right), 1.85\left(\mathrm{~m},-\mathrm{C}_{2}-\mathrm{CH}_{3}, 4 \mathrm{H}\right), 2.19\left(\mathrm{~m},-\mathrm{C}_{2}-\right.$ $\left.\mathrm{CH}_{3}, 4 \mathrm{H}\right), 2.45$ (m, $\left.\mathrm{CH}_{2}-\mathrm{C}_{2}-\mathrm{CH}_{2}, 3 \mathrm{H}\right), 2.60$ (m, $\left.\mathrm{CH}_{2}-\mathrm{CH}_{2}-\mathrm{CH}_{2}, 1 \mathrm{H}\right), 2.90$ (m, NCH, $\left.4 \mathrm{H}\right), 3.07$ 
$\left(\mathrm{m}, \mathrm{NCH}_{2}, 4 \mathrm{H}\right), 6.77-6.79$ (m, Ar, 2H), $7.48-7.49$ (m, Ar, 2H). ${ }^{11} \mathrm{~B} \mathrm{NMR}\left(\mathrm{CDCl}_{3}, 20{ }^{\circ} \mathrm{C}\right): \delta$ 24.2 (br s, FWHM = $390 \mathrm{~Hz}) .{ }^{13} \mathrm{C}\left\{{ }^{1} \mathrm{H}\right\} \mathrm{NMR}\left(\mathrm{CDCl}_{3}, 20{ }^{\circ} \mathrm{C}\right): \delta 8.8\left(-\mathrm{CH}_{2}-\mathrm{CH}_{3}\right), 24.5\left(\mathrm{~m},-\mathrm{CH}_{2}-\right.$ $\left.\mathrm{CH}_{3}\right), 26.8\left(\mathrm{~s}, \mathrm{CH}_{2}-\mathrm{CH}_{2}-\mathrm{CH}_{2}\right), 42.5\left(\mathrm{~s}, \mathrm{NCH}_{2}\right), 51.0\left(\mathrm{~s}, \mathrm{NCH}_{2}\right), 121.9$ (s, Ar), 129.7 (s, Ar), 143.8 (m, Ar). ${ }^{31} \mathrm{P}\left\{{ }^{1} \mathrm{H}\right\} \mathrm{NMR}\left(\mathrm{CDCl}_{3}, 20^{\circ} \mathrm{C}\right): \delta 61.2$ (s with doublet satellites, $\left.{ }^{1} \mathrm{JPtP}=2912 \mathrm{~Hz}\right)$.

( ${ }^{\mathrm{MeO}} \mathrm{TBDPhos}_{\mathrm{M}} \mathrm{Mo}(\mathrm{CO})_{4}$ (8). A mixture of $\mathrm{Mo}(\mathrm{CO})_{6}(1.00 \mathrm{~g}, 3.79 \mathrm{mmol})$ and ${ }^{\mathrm{MeO}} \mathrm{TBDPhos}$ $(1.23 \mathrm{~g}, 3.81 \mathrm{mmol})$ in THF $(50 \mathrm{~mL})$ was heated to reflux overnight. The resulting pale-yellow solution was evaporated to dryness under vacuum and extracted with $\mathrm{Et}_{2} \mathrm{O}(50 \mathrm{~mL})$. The paleyellow solution was allowed to cool to RT, evaporated to dryness under vacuum, and the residue was dissolved in $\mathrm{Et}_{2} \mathrm{O}(3 \mathrm{~mL})$. Layering the solution with pentane formed an off-white precipitate after 2 days. The solution was filtered through a Celite column and stored at $-30{ }^{\circ} \mathrm{C}$ to yield colorless blocks. Yield: $0.90 \mathrm{~g}(45 \%)$. Anal. Calcd for $\mathrm{C}_{14} \mathrm{H}_{24} \mathrm{BMoN}_{3} \mathrm{O}_{8} \mathrm{P}_{2}$ : C, 31.7; H, 4.56; N, 7.91. Found: $\mathrm{C}, 31.8 ; \mathrm{H}, 4.47 ; \mathrm{N}, 7.82 .{ }^{1} \mathrm{H} \mathrm{NMR}\left(\mathrm{CDCl}_{3}, 20{ }^{\circ} \mathrm{C}\right): \delta 1.79$ (quint, $\mathrm{CH}_{2}-\mathrm{CH}_{2}-\mathrm{CH}_{2}$, $4 \mathrm{H}), 2.92\left(\mathrm{t}, \mathrm{NCH}_{2}, 4 \mathrm{H}\right), 3.24\left(\mathrm{~m}, \mathrm{NCH}_{2}, 4 \mathrm{H}\right), 3.50-3.57\left(\mathrm{~m}, \mathrm{OCH}_{3}, 12 \mathrm{H}\right) .{ }^{11} \mathrm{~B} \mathrm{NMR}\left(\mathrm{CDCl}_{3}\right.$, $\left.20{ }^{\circ} \mathrm{C}\right): \delta 24.2(\mathrm{br} \mathrm{s}, \mathrm{FWHM}=320 \mathrm{~Hz}) .{ }^{13} \mathrm{C}\left\{{ }^{1} \mathrm{H}\right\} \mathrm{NMR}\left(\mathrm{CDCl}_{3}, 20{ }^{\circ} \mathrm{C}\right): \delta 26.6\left(\mathrm{~s}, \mathrm{CH}_{2}-\mathrm{CH}_{2}-\mathrm{CH}_{2}\right)$, $39.8\left(\mathrm{~s}, \mathrm{NCH}_{2}\right), 49.5\left(\mathrm{~s}, \mathrm{NCH}_{2}\right), 51.5\left(\mathrm{~s}, \mathrm{OCH}_{3}\right), 208.6(\mathrm{t}, \mathrm{CO}, J=13 \mathrm{~Hz}), 213.7$ (vt, $\mathrm{CO}, J=10$ $\mathrm{Hz}) .{ }^{31} \mathrm{P}\left\{{ }^{1} \mathrm{H}\right\} \mathrm{NMR}\left(\mathrm{CDCl}_{3}, 20{ }^{\circ} \mathrm{C}\right): \delta 165.4$ (s with sextet satellites, $\left.{ }^{1} \mathrm{~J}_{\mathrm{MoP}}=194 \mathrm{~Hz}\right) . \mathrm{MS}(\mathrm{EI}) \mathrm{m} / \mathrm{z}$ : calcd for $\mathrm{C}_{14} \mathrm{H}_{24} \mathrm{BMoN}_{3} \mathrm{O}_{8} \mathrm{P}_{2}$ 533.0186; found 533.0201. IR ( $\left.\mathrm{KBr}, \mathrm{cm}^{-1}\right)$ : $2941 \mathrm{~m}, 2889 \mathrm{~m}, 2851$ m, 2837 m, 2025 vs (CO), 1924 vs (CO), 1903 vs, (CO) 1889 vs (CO), 1507 s, 1473 m, 1463 vw, $1443 \mathrm{~m}, 1391 \mathrm{~m}, 1366 \mathrm{~m}, 1352 \mathrm{~m}, 1320 \mathrm{w}, 1297 \mathrm{w}, 1277 \mathrm{~s}, 1228 \mathrm{w}, 1207 \mathrm{~s}, 1169 \mathrm{~s}, 1121 \mathrm{w}, 1093$ m, 1065 w, 1024 vs, 938 m, 909 m, 893 m, 828 s, 788 s, 751 w, 727 s, 664 w, 645 m, 606 s, 596 $\mathrm{w}, 567 \mathrm{~m}, 546 \mathrm{~m}$.

[( $\left.\left.{ }^{\mathrm{MeO}} \mathrm{TBDPhos}^{-H}\right) \mathrm{Mo}(\mathbf{C O})_{4}\right] \mathbf{N T f}_{\mathbf{2}}\left(\mathbf{8}-\mathrm{HNTf}_{2}\right)$. To a stirring solution of $\mathbf{8}(0.10 \mathrm{~g}, 0.19 \mathrm{mmol})$ in $\mathrm{Et}_{2} \mathrm{O}(1 \mathrm{~mL})$ was added a solution of $\operatorname{HNTf}_{2}(0.053 \mathrm{~g}, 0.19 \mathrm{mmol})$ in $\mathrm{Et}_{2} \mathrm{O}(1 \mathrm{~mL})$. The solution turned pale yellow after stirring overnight and was evaporated to dryness under vacuum to yield a colorless, analytically-pure powder. Anal. Calcd for $\mathrm{C}_{16} \mathrm{H}_{25} \mathrm{BF}_{6} \mathrm{MoN}_{4} \mathrm{O}_{12} \mathrm{P}_{2} \mathrm{~S} 2$ : $\mathrm{C}, 23.7 ; \mathrm{H}, 3.10 ; \mathrm{N}$, 6.90. Found: C, 23.8; H, 3.09; N, 6.82. ${ }^{1} \mathrm{H} \mathrm{NMR}\left(\mathrm{CDCl}_{3}, 20{ }^{\circ} \mathrm{C}\right): \delta 1.97(\mathrm{~m}, 2 \mathrm{H}), 2.15(\mathrm{~m}, 2 \mathrm{H})$, $2.98(\mathrm{~m}, 2 \mathrm{H}), 3.31(\mathrm{~m}, 2 \mathrm{H}), 3.54-3.73(\mathrm{~m}, 16 \mathrm{H}), 7.08$ (br s, NH, 1H). ${ }^{11} \mathrm{~B} \mathrm{NMR}\left(\mathrm{CDCl}_{3}, 20{ }^{\circ} \mathrm{C}\right)$ : $\delta 27.8$ (br s, FWHM = 400 Hz). ${ }^{19} \mathrm{~F} \mathrm{NMR}\left(\mathrm{CDCl}_{3}, 20{ }^{\circ} \mathrm{C}\right): \delta-79.3(\mathrm{~s}) .{ }^{31} \mathrm{P}\left\{{ }^{1} \mathrm{H}\right\} \mathrm{NMR}\left(\mathrm{CDCl}_{3}, 20\right.$ $\left.{ }^{\circ} \mathrm{C}\right): \delta 167.0(\mathrm{~s}) . \mathrm{IR}\left(\mathrm{KBr}, \mathrm{cm}^{-1}\right): 3116 \mathrm{~m}(\mathrm{NH}), 2982 \mathrm{w}, 2949 \mathrm{~m}, 2847 \mathrm{w}, 2042 \mathrm{vs}(\mathrm{CO}), 1947 \mathrm{vw}$ (CO), 1915 vs (CO), 1479 m, 1449 m, 1435 m, 1345 s, 1322 s, 1302 vw, 1275 vw, 1262 vw, 1241 vw, 1193 s, 1135 m, 1099 m, 1059 s, 1029 s, 929 w, 915 vw, 890 vw, 820 vw, 764 s, 741 s, 669 vw, $653 \mathrm{w}, 611 \mathrm{~m}, 594 \mathrm{~m}, 570 \mathrm{~m}, 539 \mathrm{w}, 508 \mathrm{~m}$.

( $\left.\left.{ }^{\mathrm{MeO}}{ }_{\mathrm{TBDPhos}} \mathrm{MeOH}\right) \mathrm{Mo}(\mathrm{CO})_{4} \mathbf{( 8 - M e O H}\right)$. To a stirring solution of 8 (0.10 g, $\left.0.19 \mathrm{mmol}\right)$ in $\mathrm{Et}_{2} \mathrm{O}(1 \mathrm{~mL})$ was added $\mathrm{MeOH}(0.5 \mathrm{~mL})$ and $\mathrm{NEt}_{3}(0.1 \mathrm{~mL})$ in air. The colorless solution was stirred overnight. Colorless blocks were obtained by slowly allowing the solution to evaporate in air. The resulting crystals were isolated by filtration and washed with hexane $(10 \mathrm{~mL})$. Yield: 0.089 g (84\%). Anal. Calcd for $\mathrm{C}_{15} \mathrm{H}_{28} \mathrm{BMoN}_{3} \mathrm{O}_{9} \mathrm{P}_{2}: \mathrm{C}, 32.0 ; \mathrm{H}, 5.01 ; \mathrm{N}, 7.46$. Found: $\mathrm{C}, 32.0 ; \mathrm{H}, 4.89$; $\mathrm{N}, 7.74 .{ }^{1} \mathrm{H} \mathrm{NMR}\left(\mathrm{CDCl}_{3}, 20^{\circ} \mathrm{C}\right): \delta 1.61(\mathrm{~m}, 2 \mathrm{H}), 1.79(\mathrm{~m}, 2 \mathrm{H}), 2.80(\mathrm{~m}, 4 \mathrm{H}), 3.06(\mathrm{~m}, 3 \mathrm{H}), 3.15$ $\left(\mathrm{s}, \mathrm{BOCH}_{3}, 3 \mathrm{H}\right), 3.43-3.59\left(\mathrm{~m}, \mathrm{P}\left(\mathrm{OCH}_{3}\right)_{2} 12 \mathrm{H}\right), 3.71(\mathrm{~m}, 2 \mathrm{H}) .{ }^{11} \mathrm{~B} \mathrm{NMR}\left(\mathrm{CDCl}_{3}, 20{ }^{\circ} \mathrm{C}\right): \delta 2.7$ (br s, FWHM = $80 \mathrm{~Hz}) \cdot{ }^{13} \mathrm{C}\left\{{ }^{1} \mathrm{H}\right\} \mathrm{NMR}\left(\mathrm{CDCl}_{3}, 20{ }^{\circ} \mathrm{C}\right): \delta 28.3\left(\mathrm{~s}, \mathrm{CH}_{2}-\underline{\mathrm{CH}}_{2}-\mathrm{CH}_{2}\right), 38.3\left(\mathrm{vt}, \mathrm{NCH}_{2}\right.$, $J=2 \mathrm{~Hz}), 46.9\left(\mathrm{~s}, \mathrm{NCH}_{2}\right), 51.2\left(\mathrm{~s}, \mathrm{P}\left(\mathrm{OCH}_{3}\right)_{2}\right), 51.3\left(\mathrm{~s}, \mathrm{P}\left(\mathrm{OCH}_{3}\right)_{2}\right), 51.9\left(\mathrm{~s}, \mathrm{BOCH}_{3}\right), 209.5(\mathrm{t}, \mathrm{CO}$, $J=13 \mathrm{~Hz}), 210.3(\mathrm{t}, \mathrm{CO}, J=13 \mathrm{~Hz}), 214.5$ (vt, CO, $J=10 \mathrm{~Hz}) \cdot{ }^{31} \mathrm{P}\left\{{ }^{1} \mathrm{H}\right\} \mathrm{NMR}\left(\mathrm{CDCl}_{3}, 20{ }^{\circ} \mathrm{C}\right): \delta$ 165.3 (s with sextet satellites, $\left.{ }^{1} J_{\mathrm{MoP}}=192 \mathrm{~Hz}\right) . \mathrm{IR}\left(\mathrm{KBr}, \mathrm{cm}^{-1}\right): 3266 \mathrm{~m}(\mathrm{NH}), 2970 \mathrm{~m}, 2956 \mathrm{~m}$, 2940 m, 2885 m, 2832 m, 2820 m, 2018 vs (CO), 1933 vs (CO), 1909 vs (CO), 1872 vs (CO), 1463 m, 1438 m, 1404 w, 1365 m, 1343 w, 1295 m, 1242 m, 1194 m, 1180 vw, 1141 s, 1108 s, 
1092 s, 1081 s, 1060 s, 1038 s, 1026 s, 1010 s, 963 m, 951 vw, 918 m, 874 m, 832 w, 791 vw, 779 m, 743 m, 728 m, 709 m, 676 vw, 661 vw, 647 vw, 619 s, 599 s, 566 m, 535 vw, 527 m, 515 vw, $501 \mathrm{vw}$.

Crystallographic studies. Single crystals were obtained from $\mathrm{CH}_{2} \mathrm{Cl}_{2} / \mathrm{Et}_{2} \mathrm{O}(\mathbf{1}, \mathbf{2}, 5$ and 6),

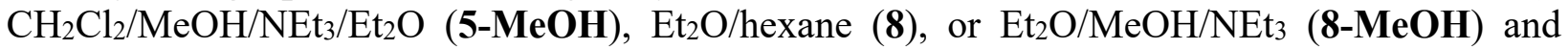
mounted on a MiTeGen micromount using ParatoneN oil in air. The data collection, structural solution, and refinement were carried out as reported previously. ${ }^{3}$ Briefly, the structures were solved with Direct Methods (SHELXT) ${ }^{4}$ and the positions of the non-hydrogen atoms were confirmed by subsequent least-squares refinement (SHELXL). ${ }^{5}$ The positional disorder of the $\mathrm{P}(\mathrm{OMe})_{2}$ groups in 5-MeOH was modeled by refining each atom over two positions and setting the sum of the site occupancy factor equal to one. The disordered TBD subunit in $\mathbf{6}$ was modeled similarly. The positions of all hydrogen atoms were idealized and allowed to ride on the attached atom. The final refinement included anisotropic temperature factors on all non-hydrogen atoms. Structure solution and refinement were performed with $\mathrm{Olex}^{2}$ and publication figures were generated with Olex ${ }^{2}$ or Mercury. ${ }^{6}$

DFT Calculations. Geometry optimizations were performed using the M06-L density functional and the def2-SVP basis set for main group elements while the def2-TZVP and its corresponding ECP were used for $\mathrm{Pt} .{ }^{7}$ Calculations were performed at room temperature and using $\mathrm{CH}_{2} \mathrm{Cl}_{2}$ as the solvent. All DFT calculations were performed using the Gaussian 16 software package. We also tested the choice of the meta-GGA M06-L functional against the hybrid B3LYP functional. ${ }^{8}$ For the B3LYP functional, the choice of basis set and ECP remain the same, however a dispersion correction (Grimme's D3 correction with Becke-Johnson damping) ${ }^{9}$ was incorporated. Free energies of reaction for ( $\left.{ }^{\mathrm{R}} \mathrm{TBDPhos}\right) \mathrm{Pt}\left(\mathrm{S}_{2} \mathrm{C}_{6} \mathrm{H}_{4}\right)$ with $\mathrm{CH}_{3} \mathrm{OH}$ were computed with both M06L and B3LYP functionals. After computing geometries from different initial guesses, we chose the most stable conformer for reactant and product. The supplemental file optimized-geometries.xyz contains the computed Cartesian coordinates of the molecules reported in this study. The file may be opened as a text file to read the coordinates, or opened directly by a molecular modeling program such as Mercury (version 3.3 or later, http://www.ccdc.cam.ac.uk/pages/Home.aspx $)^{6 \mathrm{~b}}$ for visualization and analysis. 
Table S1. Calculated $\Delta \mathrm{G}$ of reaction computed using the M06-L and B3LYP functionals for $\left({ }^{\mathrm{R}} \mathrm{TBDPhos}\right) \mathrm{Pt}\left(\mathrm{S}_{2} \mathrm{C}_{6} \mathrm{H}_{4}\right)+\mathrm{MeOH} \rightarrow\left({ }^{\mathrm{R}} \mathrm{TBDPhos}-\mathrm{MeOH}\right) \mathrm{Pt}\left(\mathrm{S}_{2} \mathrm{C}_{6} \mathrm{H}_{4}\right)$.

\begin{tabular}{lcc}
\hline $\mathbf{R}$ & M06-L & B3LYP \\
\hline OMe & -5.79 & -9.01 \\
OEt & -3.98 & -8.39 \\
$\mathrm{Me}$ & -1.83 & -6.09 \\
$\mathrm{Ph}$ & -0.93 & -4.01 \\
$\mathrm{Et}$ & 2.46 & 0.74 \\
${ }^{i} \mathrm{Pr}$ & 6.59 & 2.58 \\
\hline
\end{tabular}

Table S2. DFT calculated Gibbs free energies of $\left({ }^{\mathrm{R}} \mathrm{TBDPhos}\right) \mathrm{Pt}\left(\mathrm{S}_{2} \mathrm{C}_{6} \mathrm{H}_{4}\right)$ (reactant) and ( $\left.{ }^{\mathrm{R}} \mathrm{TBDPhos}-\mathrm{MeOH}\right) \mathrm{Pt}\left(\mathrm{S}_{2} \mathrm{C}_{6} \mathrm{H}_{4}\right)$ (product) using the M06L functional. Calculations for Product 1 and Product 2 were performed using different starting orientations of the OMe groups on the TBD backbone. Product 1 started with OMe pointing towards Pt (as observed in the XRD structure of 5-MeOH) whereas Product 2 started with OMe pointing away from Pt.

\begin{tabular}{|c|c|c|c|c|c|}
\hline \multirow[b]{2}{*}{$\mathbf{R}=$} & \multirow[b]{2}{*}{ Complex } & \multicolumn{4}{|c|}{ M06L } \\
\hline & & SCF (a.u) & Total Energy (a.u) & Gibbs Free energy (a.u) & $\Delta \mathbf{G}(\mathrm{kcal} / \mathrm{mol})^{\dagger}$ \\
\hline \multirow[t]{3}{*}{ Me } & Reactant & -2413.3690 & -2412.9084 & -2412.9957 & \\
\hline & Product 1 & -2529.0151 & -2528.4957 & -2528.5877 & -1.83 \\
\hline & Product $2 *$ & -2529.0151 & -2528.4957 & -2528.5877 & -1.84 \\
\hline \multirow[t]{3}{*}{ OMe } & Reactant & -2714.0174 & -2713.5305 & -2713.6300 & \\
\hline & Product 1 & -2829.6688 & -2829.1231 & -2829.2283 & -5.79 \\
\hline & Product $2^{*}$ & -2829.6688 & -2829.1240 & -2829.2254 & -3.95 \\
\hline \multirow[t]{3}{*}{${ }^{i} \mathbf{P r}$} & Reactant & -2727.5917 & -2726.8918 & -2726.9969 & \\
\hline & Product 1 & -2843.2242 & -2842.4655 & -2842.5755 & 6.59 \\
\hline & Product 2 & -2843.2209 & -2842.4629 & -2842.5726 & 8.39 \\
\hline \multirow[t]{3}{*}{$\mathbf{P h}$} & Reactant & -3179.6944 & -3179.0065 & -3179.1235 & \\
\hline & Product 1 & -3295.3383 & -3294.5916 & -3294.7141 & -0.93 \\
\hline & Product 2 & -3295.3278 & -3294.5812 & -3294.7030 & 6.02 \\
\hline \multirow[t]{3}{*}{ OEt } & Reactant & -2871.1665 & -2870.5617 & -2870.6733 & \\
\hline & Product 1 & -2986.8171 & -2986.1531 & -2986.2688 & -3.98 \\
\hline & Product $2^{*}$ & -2986.8171 & -2986.1531 & -2986.2687 & -3.96 \\
\hline \multirow[t]{4}{*}{ Et } & Reactant & -2570.4781 & -2569.8967 & -2569.9939 & \\
\hline & Product 1 & -2686.1162 & -2685.4759 & -2685.5791 & 2.46 \\
\hline & Product 2 & -2686.1048 & -2685.46415 & -2685.56658 & 10.34 \\
\hline & МeOH & -2413.3690 & -2412.9084 & -115.5891 & \\
\hline
\end{tabular}

*Product 2 converges to a structure that is similar to Product 1. $\dagger \Delta \mathrm{G}=\mathrm{G}$ (Product) $-\mathrm{G}$ (Reactant $+\mathrm{MeOH}$ ) 

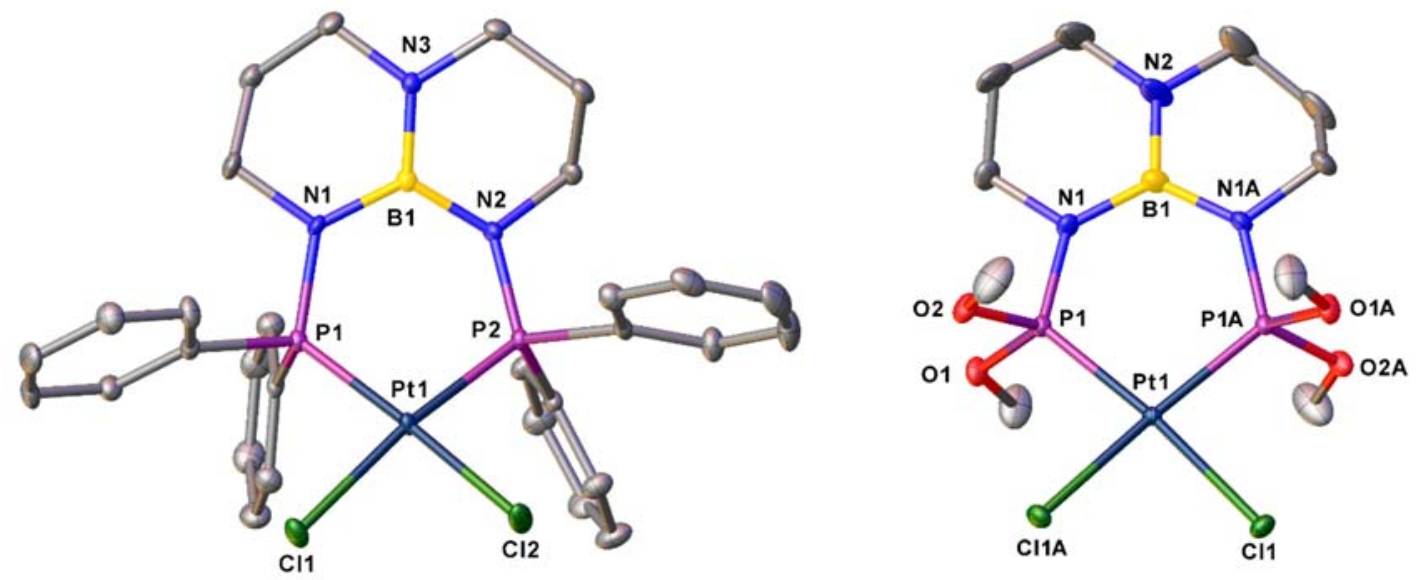

Figure S1. Molecular structures of ( $\left.{ }^{\mathrm{Ph}} \mathrm{TBDPhos}\right) \mathrm{PtCl}_{2}$ (1; left) and ( $\left.{ }^{\mathrm{MeO}} \mathrm{TBDPhos}\right) \mathrm{PtCl}_{2}$ (2; right) with thermal ellipsoids drawn at the $35 \%$ probability level. Co-crystallized solvent molecules and hydrogen atoms were omitted from the figure.

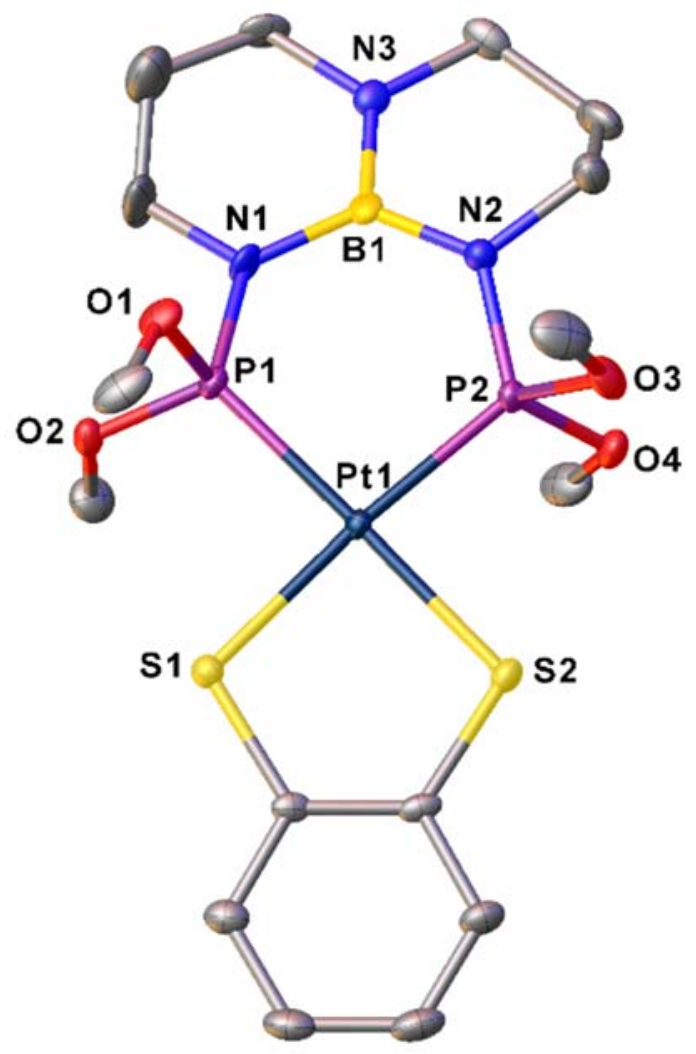

Figure S2. Molecular structure of ( $\left.{ }^{\mathrm{MeO}} \mathrm{TBDPhos}\right) \mathrm{Pt}\left(\mathrm{S}_{2} \mathrm{C}_{6} \mathrm{H}_{4}\right)(5)$ with thermal ellipsoids drawn at the $35 \%$ probability level. Hydrogen atoms were omitted from the figure. 


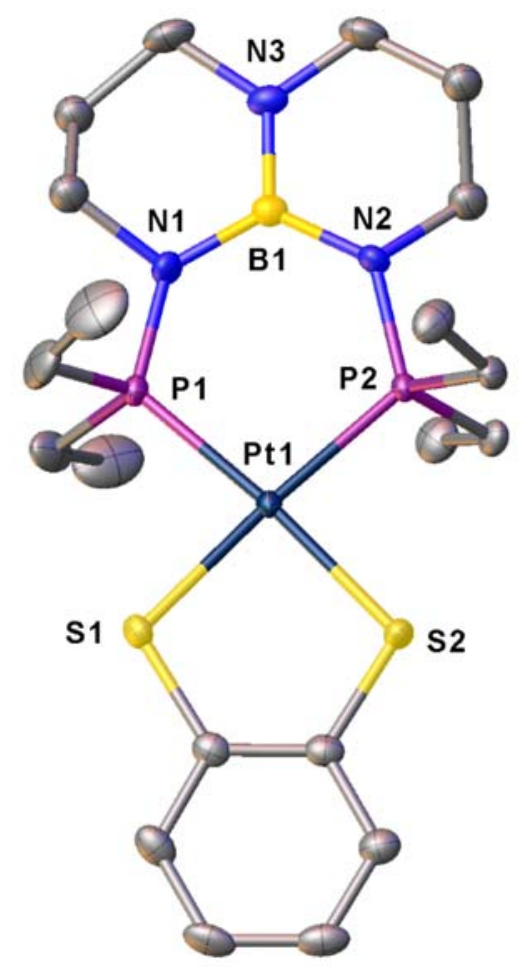

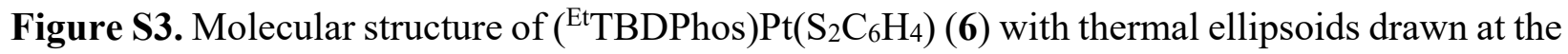
$35 \%$ probability level. Hydrogen atoms and disordered fragments were omitted from the figure.

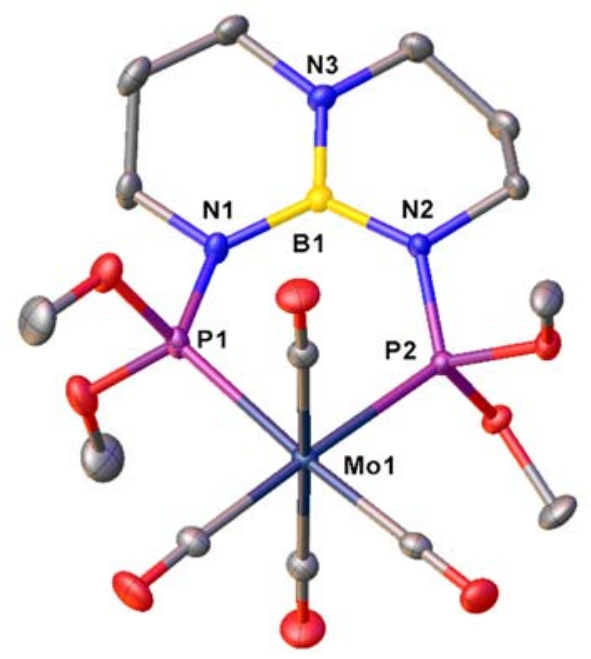

Figure S4. Molecular structure of $\left({ }^{\mathrm{MeO}} \mathrm{TBDPhos}\right) \mathrm{Mo}(\mathrm{CO})_{4}(\mathbf{8})$ with thermal ellipsoids drawn at the $35 \%$ probability level. Hydrogen atoms were omitted from the figure. 


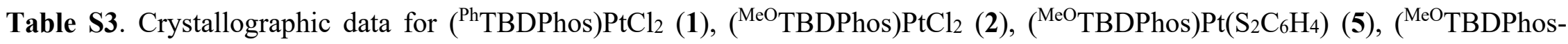
$\mathrm{MeOH}) \mathrm{Pt}\left(\mathrm{S}_{2} \mathrm{C}_{6} \mathrm{H}_{4}\right)$ (5-MeOH), ( $\left.{ }^{\mathrm{E} t} \mathrm{TBDPhos}\right) \mathrm{Pt}\left(\mathrm{S}_{2} \mathrm{C}_{6} \mathrm{H}_{4}\right)$ (6), ( ${ }^{\mathrm{MeO}} \mathrm{TBDPhos}^{\mathrm{T}} \mathrm{Mo}(\mathrm{CO}) 4$ (8), and ( $\left.{ }^{\mathrm{MeO}} \mathrm{TBDPhos}-\mathrm{MeOH}\right) \mathrm{Mo}(\mathrm{CO}) 4($ 8$\mathrm{MeOH})$.

\begin{tabular}{|c|c|c|c|c|c|c|c|}
\hline & 1 & 2 & 5 & 5-МеОН & 6 & 8 & 8-МеОН \\
\hline formula & $\mathrm{C}_{32} \mathrm{H}_{36} \mathrm{BCl}_{6} \mathrm{~N}_{3} \mathrm{P}_{2} \mathrm{Pt}$ & $\mathrm{C}_{10} \mathrm{H}_{24} \mathrm{BCl}_{2} \mathrm{~N}_{3} \mathrm{O}_{4} \mathrm{P}_{2} \mathrm{Pt}$ & $\mathrm{C}_{16} \mathrm{H}_{28} \mathrm{BN}_{3} \mathrm{O}_{4} \mathrm{P}_{2} \mathrm{PtS}_{2}$ & $\mathrm{C}_{17} \mathrm{H}_{32} \mathrm{BN}_{3} \mathrm{O}_{5} \mathrm{P}_{2} \mathrm{PtS}_{2}$ & $\mathrm{C}_{20} \mathrm{H}_{36} \mathrm{BN}_{3} \mathrm{P}_{2} \mathrm{PtS}_{2}$ & $\mathrm{C}_{14} \mathrm{H}_{24} \mathrm{BMoN}_{3} \mathrm{O}_{8} \mathrm{P}_{2}$ & $\mathrm{C}_{15} \mathrm{H}_{28} \mathrm{BMoN}_{3} \mathrm{O}_{9} \mathrm{P}_{2}$ \\
\hline $\mathrm{FW}\left(\mathrm{g} \mathrm{mol}^{-1}\right)$ & 943.18 & 589.06 & 658.38 & 690.41 & 640.48 & 531.05 & 563.09 \\
\hline crystal system & Triclinic & Orthorhombic & Orthorhombic & Monoclinic & Monoclinic & Monoclinic & Triclinic \\
\hline space group & $\mathrm{P}-1$ & Pbcn & $\mathrm{P} 2{ }_{1} 2_{1} 2_{1}$ & $\mathrm{Cc}$ & $\mathrm{C} 2 / \mathrm{c}$ & $\mathrm{P} 21 / \mathrm{n}$ & $\mathrm{P}-1$ \\
\hline $\mathrm{a}(\AA)$ & $9.4060(9)$ & $14.9159(15)$ & $9.0210(9)$ & $15.9347(16)$ & $18.4280(18)$ & $10.4009(10)$ & $9.2443(9)$ \\
\hline $\mathrm{b}(\AA)$ & $12.2212(12)$ & $11.3340(11)$ & $9.4970(9)$ & $10.2361(10)$ & $17.5106(18)$ & $13.3405(13)$ & $10.2295(10)$ \\
\hline c $(\AA)$ & $16.9381(17)$ & $11.3449(11)$ & $26.448(2)$ & $14.7399(15)$ & $17.6481(18)$ & $15.7314(16)$ & $13.0524(13)$ \\
\hline$\alpha(\operatorname{deg})$ & $88.129(5)$ & 90 & 90 & 90 & 90 & 90 & $97.427(5)$ \\
\hline$\beta(\operatorname{deg})$ & $76.895(5)$ & 90 & 90 & $92.306(5)$ & $117.543(5)$ & $96.242(5)$ & $104.798(5)$ \\
\hline$\gamma(\mathrm{deg})$ & $72.751(5)$ & 90 & 90 & 90 & 90 & 90 & $100.202(5)$ \\
\hline volume $\left(\AA^{3}\right)$ & $1809.8(3)$ & 1917.9(3) & $2265.9(4)$ & $2402.3(4)$ & $5049.4(9)$ & $2169.8(4)$ & $1154.6(2)$ \\
\hline Z & 2 & 4 & 4 & 4 & 8 & 4 & 2 \\
\hline$\rho_{\text {calc }}\left(\mathrm{g} \mathrm{cm}^{-3}\right)$ & 1.731 & 2.040 & 1.930 & 1.909 & 1.711 & 1.626 & 1.620 \\
\hline$\mu\left(\mathrm{mm}^{-1}\right)$ & 4.436 & 7.779 & 6.546 & 6.182 & 5.862 & 0.797 & 0.757 \\
\hline $\mathrm{F}(000)$ & 928 & 1136 & 1288 & 1360 & 2576 & 1080 & 576 \\
\hline$\theta$ range (deg) & $2.33 / 26.49$ & $2.73 / 27.89$ & $2.39 / 27.91$ & $2.56 / 26.43$ & $2.56 / 26.38$ & $2.24 / 27.82$ & $2.34 / 28.02$ \\
\hline $\mathrm{R}$ (int) & 0.0400 & 0.0230 & 0.0375 & 0.0284 & 0.0352 & 0.0281 & 0.0249 \\
\hline data/restraints/parameters & $7446 / 0 / 406$ & $2261 / 0 / 108$ & $5380 / 0 / 267$ & $4021 / 30 / 278$ & $6248 / 12 / 261$ & $5155 / 0 / 266$ & $5086 / 0 / 286$ \\
\hline GOF & 1.038 & 1.146 & 1.105 & 1.002 & 1.049 & 1.039 & 1.064 \\
\hline $\mathrm{R}_{1}[I>2 \sigma(I)]^{\mathrm{a}}$ & 0.0260 & 0.0199 & 0.0254 & 0.0231 & 0.0203 & 0.0224 & 0.0234 \\
\hline $\mathrm{wR}_{2}$ (all data) ${ }^{\mathrm{b}}$ & 0.0528 & 0.0323 & 0.0486 & 0.0449 & 0.0456 & 0.0544 & 0.0537 \\
\hline Ext. Coeff & - & - & - & - & - & - & - \\
\hline Largest Peak/Hole $\left(\mathrm{e} \cdot \AA^{-3}\right)$ & $1.560 /-1.717$ & $0.313 /-0.803$ & $0.961 /-1.131$ & $0.666 /-0.905$ & $0.764 /-0.804$ & $0.928 /-0.506$ & $0.358 /-0.357$ \\
\hline Temp (K) & $190(2)$ & $150(2)$ & $150(2)$ & $150(2)$ & $150(2)$ & $150(2)$ & $150(2)$ \\
\hline
\end{tabular}

${ }^{\mathrm{a}} \mathrm{R}_{1}=\sum\left|F_{o}\right|-\left|F_{c}\right||/| \sum\left|F_{o}\right|$ for reflections with $F_{o}^{2}>2 \sigma\left(F_{o}^{2}\right)$.

${ }^{b_{w R}}=\left[\sum w\left(F_{o}^{2}-F_{c}^{2}\right)^{2} / \sum\left(F_{o}^{2}\right)^{2}\right]^{1 / 2}$ for all reflections. 


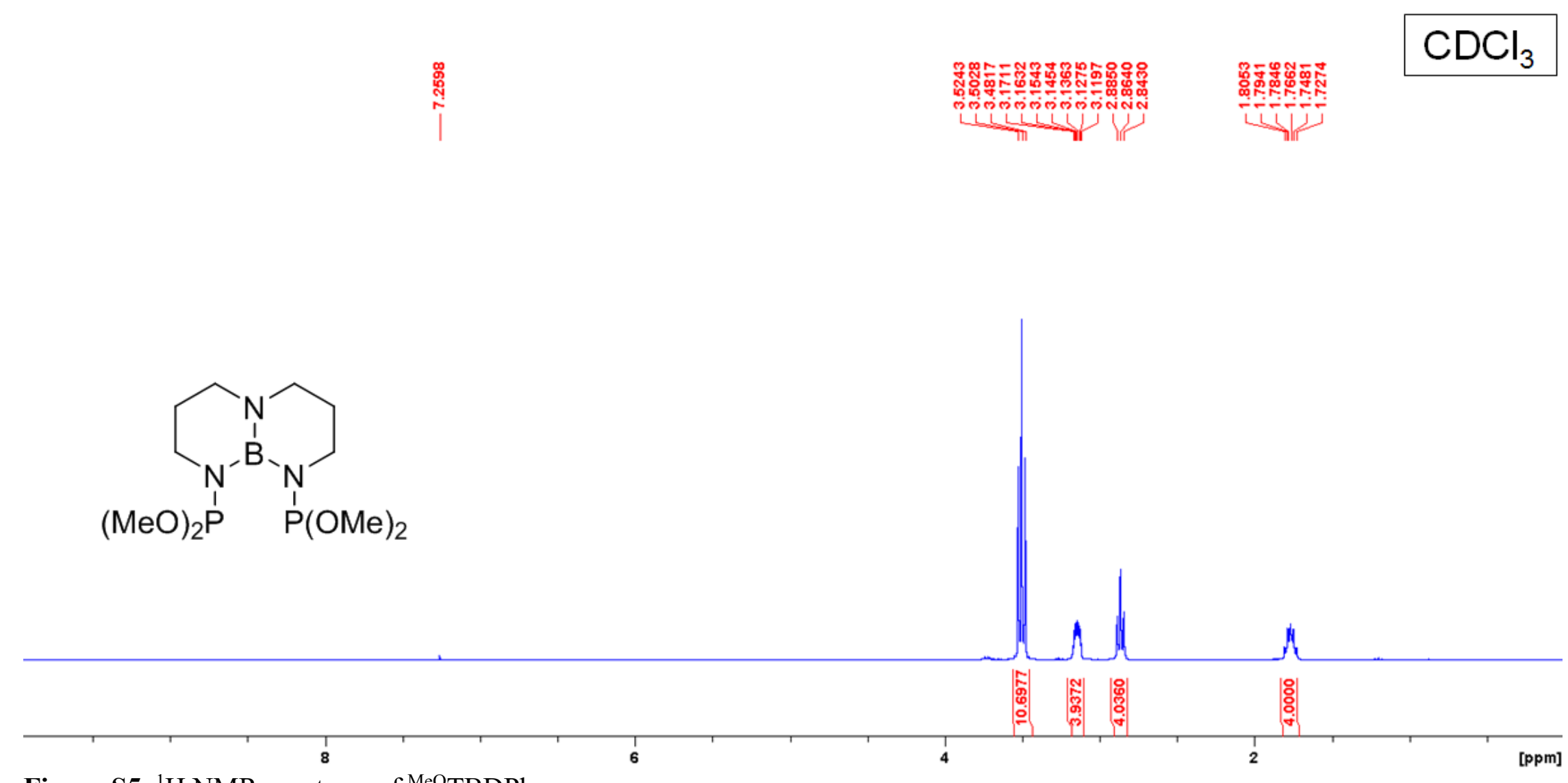

Figure S5. ${ }^{1} \mathrm{H}$ NMR spectrum of ${ }^{\mathrm{MeO}} \mathrm{TBDPhos}$. 


\section{$\mathrm{CDCl}_{3}$}

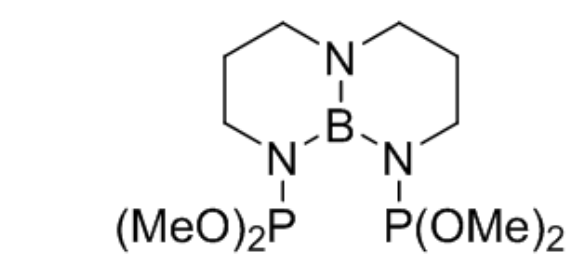

Figure S6. ${ }^{11} \mathrm{~B}$ NMR spectrum of ${ }^{\mathrm{MeO}} \mathrm{TBDPhos}$. 


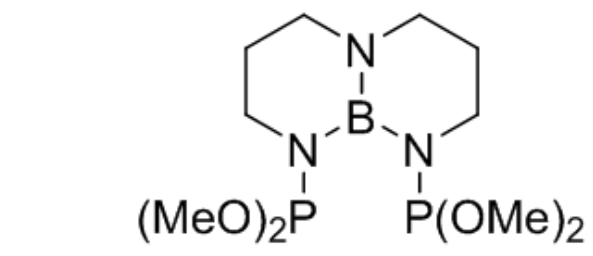




\section{$\mathrm{CDCl}_{3}$}

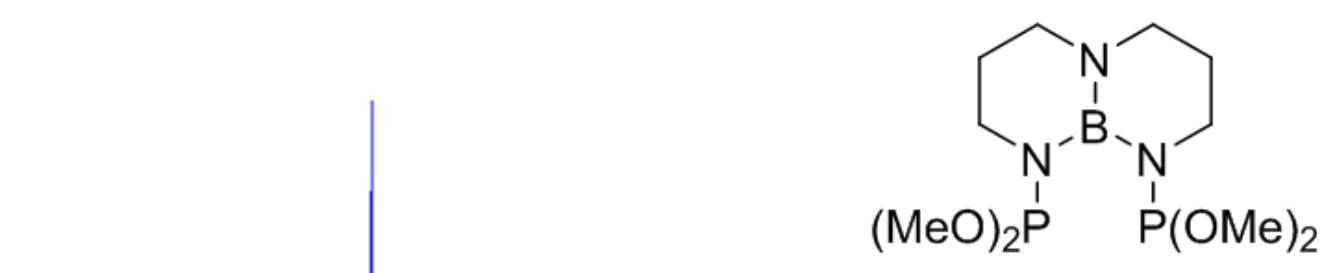

Figure S8. ${ }^{31} \mathrm{P}$ NMR spectrum of ${ }^{\mathrm{MeO}}$ TBDPhos. 


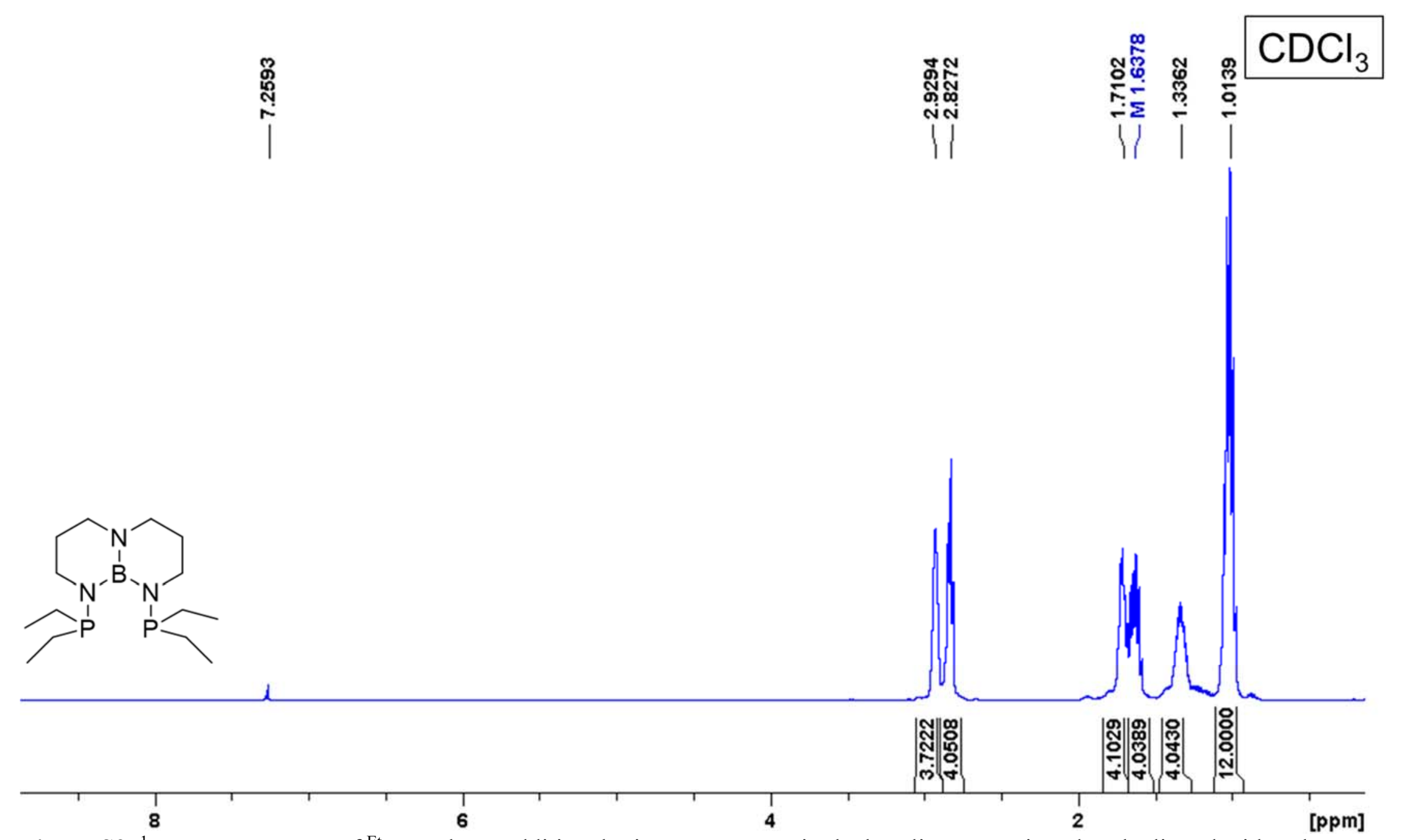

Figure S9. ${ }^{1} \mathrm{H}$ NMR spectrum of ${ }^{\mathrm{Et}} \mathrm{TBDPhos}$. Additional minor resonances in the baseline are assigned to the ligand with only one PEt 2 arm, which is a minor impurity that co-crystallizes with EtTBDPhos. 


\section{$\mathrm{CDCl}_{3}$}

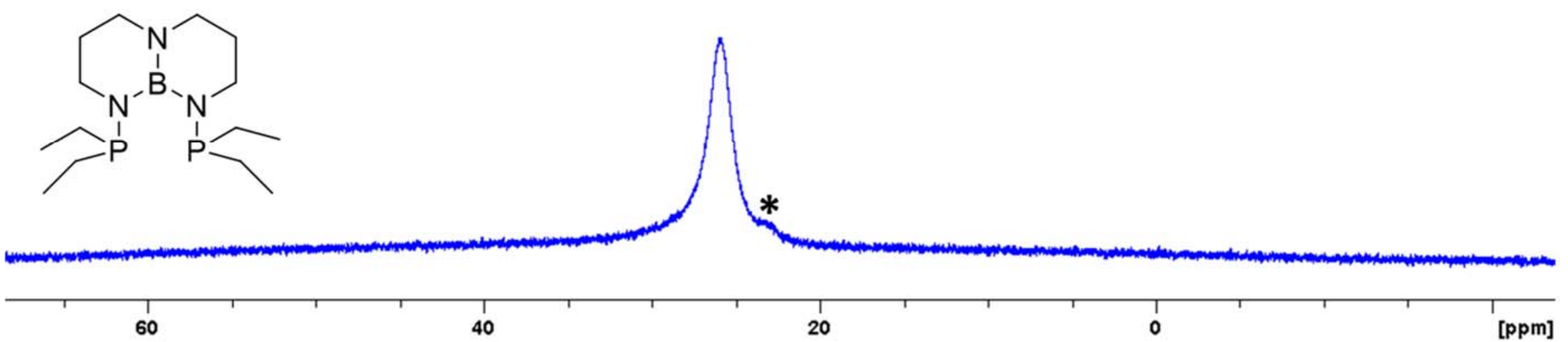

Figure S10. ${ }^{11} \mathrm{~B}$ NMR spectrum of ${ }^{\mathrm{Et}} \mathrm{TBDPhos}$. The * indicates the resonance assigned to the ligand with only one PEt 2 arm, which is a minor impurity that co-crystallizes with ${ }^{\mathrm{Et}} \mathrm{TBDPhos}$. 


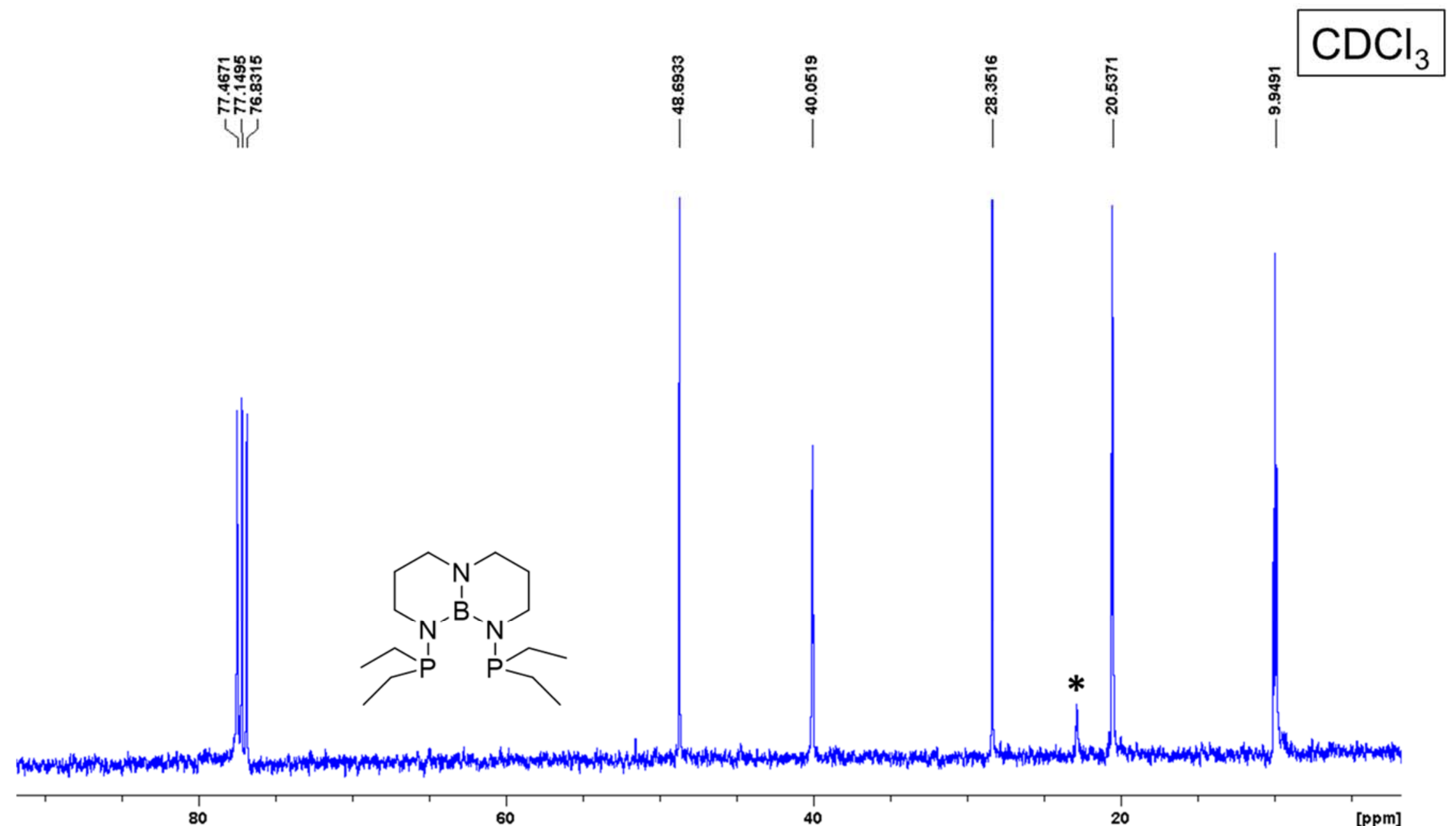

Figure S11. ${ }^{13} \mathrm{C}$ NMR spectrum of EtTBDPhos. The * indicates the minor resonance assigned to the ligand with only one PEt 2 arm, which is an impurity that co-crystallizes with ${ }^{\mathrm{Et}} \mathrm{TBDPhos}$. 
$\mathrm{CDCl}_{3}$

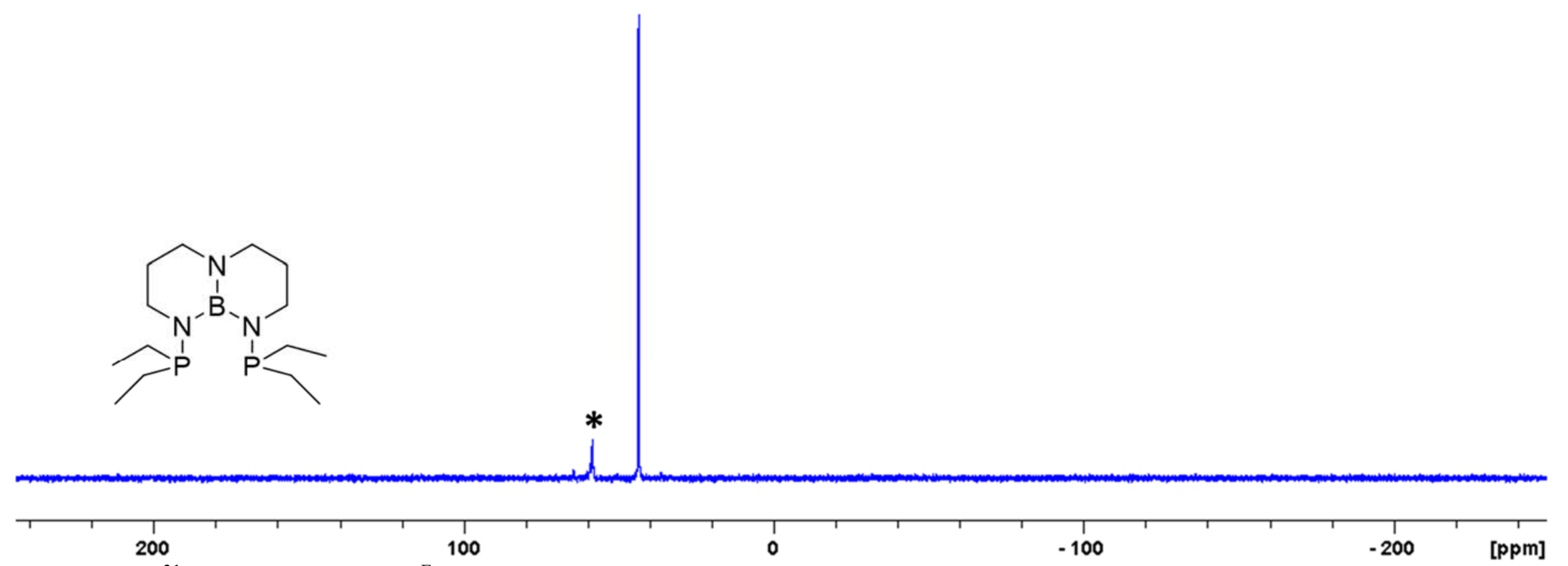

Figure S12. ${ }^{31} \mathrm{P}$ NMR spectrum of EtTBDPhos. The * indicates the minor resonance assigned to the ligand with only one PEt 2 arm, which is an impurity that co-crystallizes with ${ }^{\mathrm{Et}} \mathrm{TBDPhos}$. 


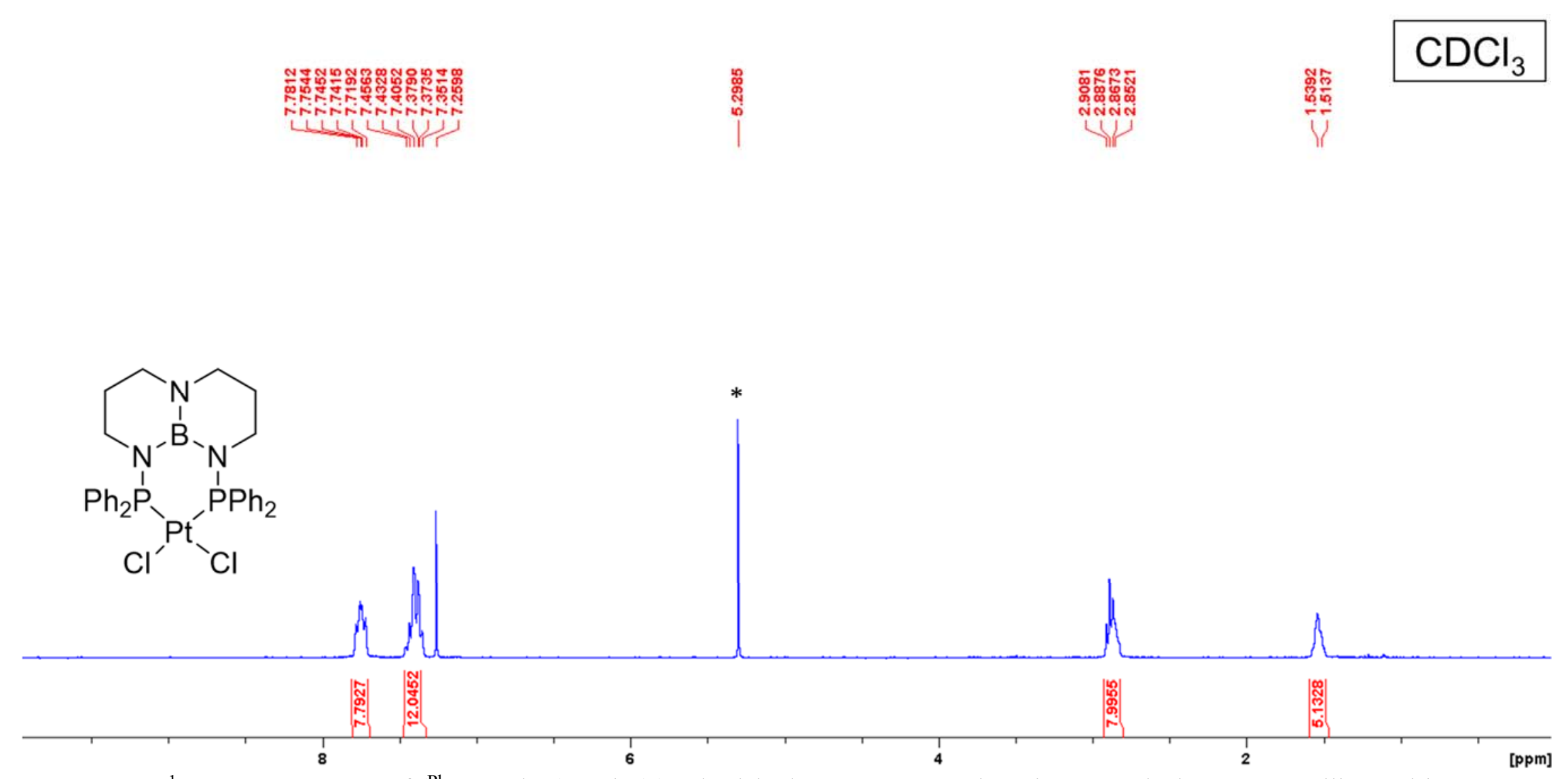

Figure S13. ${ }^{1} \mathrm{H}$ NMR spectrum of ( $\left.{ }^{\mathrm{Ph}} \mathrm{TBDPhos}\right) \mathrm{PtCl}_{2}(\mathbf{1})$. The $*$ is the resonance assigned to $\mathrm{CH}_{2} \mathrm{Cl}_{2}$ that co-crystallizes with 1 . 


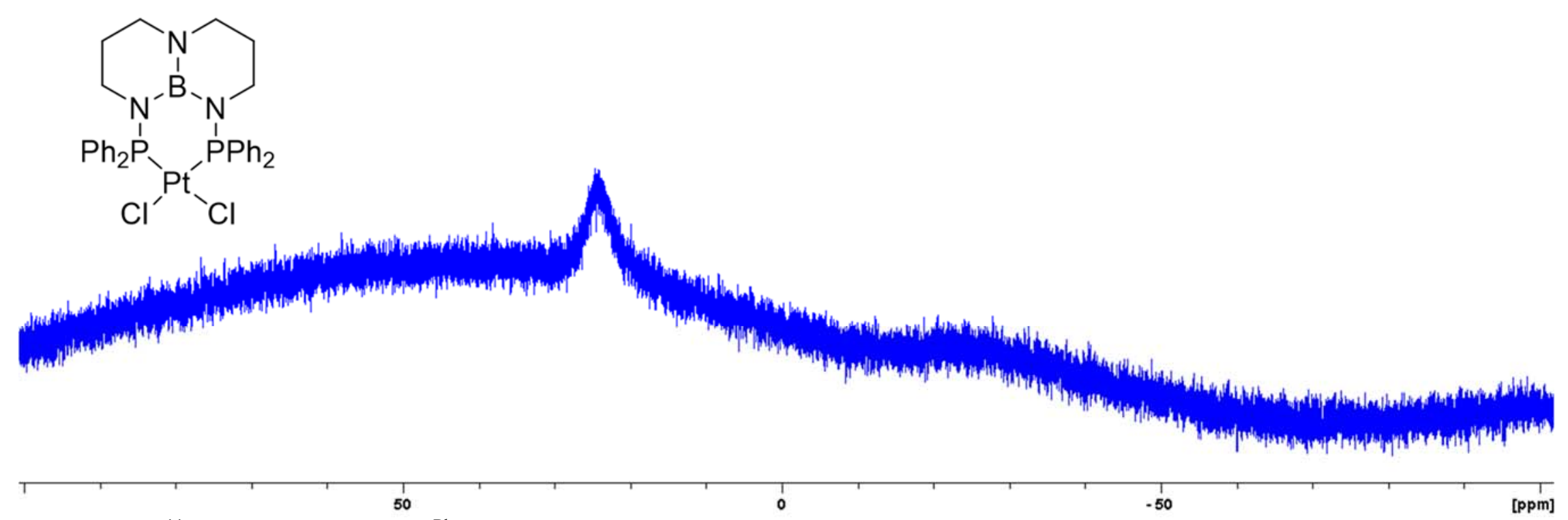

Figure S14. ${ }^{11} \mathrm{~B}$ NMR spectrum of $\left({ }^{\mathrm{Ph}} \mathrm{TBDPhos}\right) \mathrm{PtCl}_{2}$ (1). 


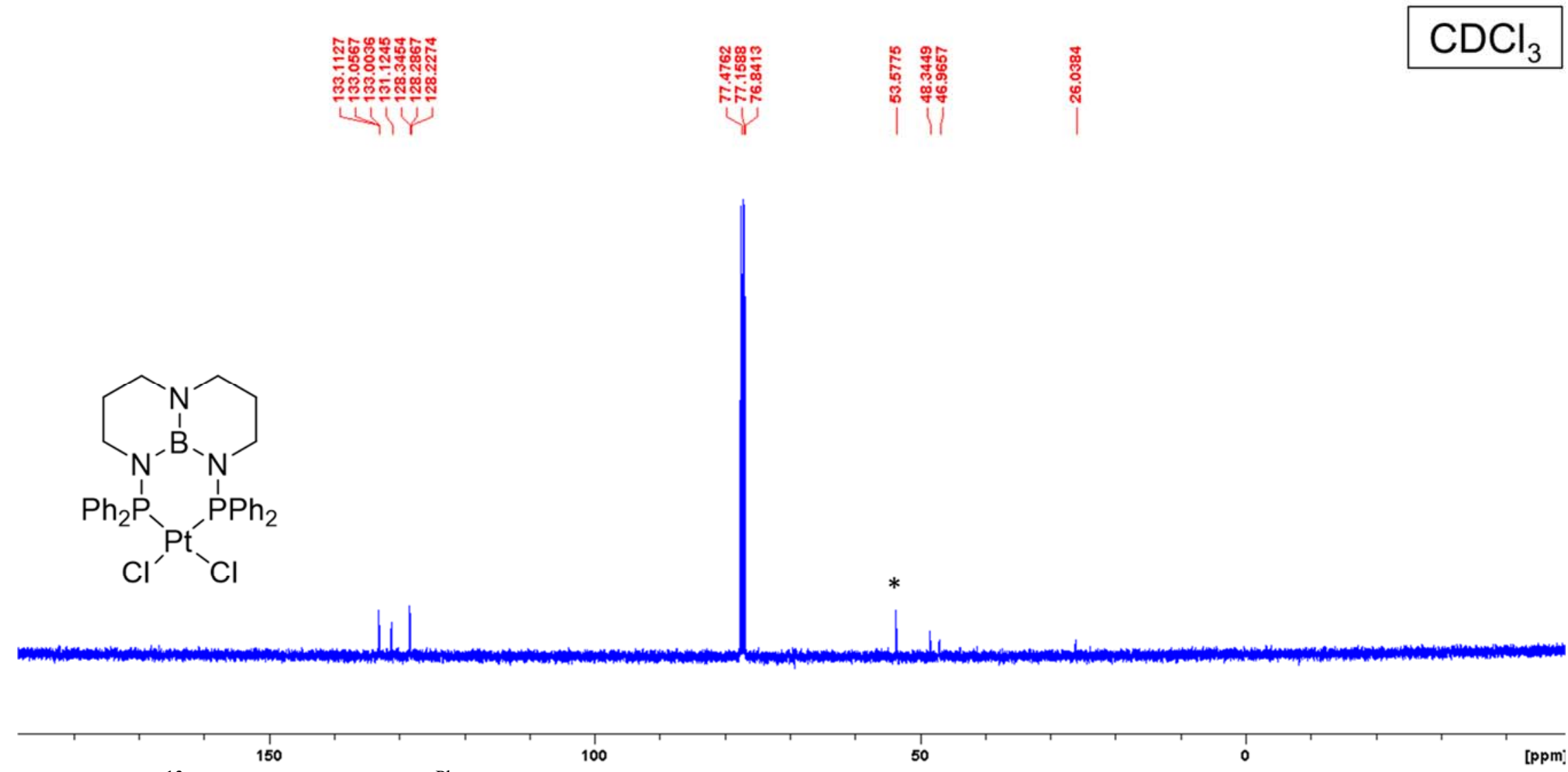

Figure S15. ${ }^{13} \mathrm{C}$ NMR spectrum of $\left({ }^{\mathrm{Ph}} \mathrm{TBDPhos}\right) \mathrm{PtCl}_{2}(\mathbf{1})$. The * is the resonance assigned to $\mathrm{CH}_{2} \mathrm{Cl}_{2}$ that co-crystallizes with 1. 


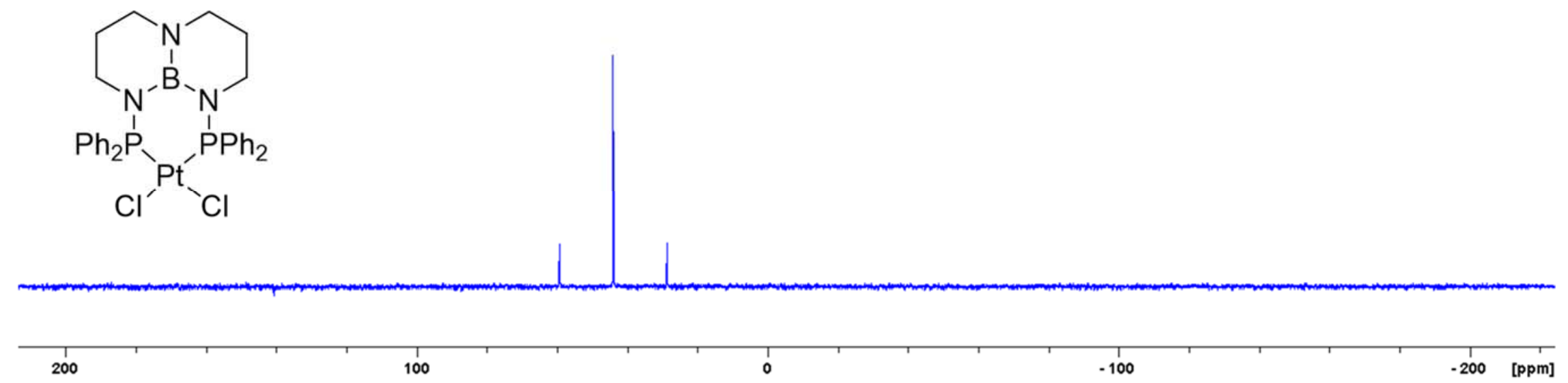

Figure S16. ${ }^{31} \mathrm{P}$ NMR spectrum of ( $\left.{ }^{\mathrm{Ph}} \mathrm{TBDPhos}\right) \mathrm{PtCl}_{2}$ (1). 


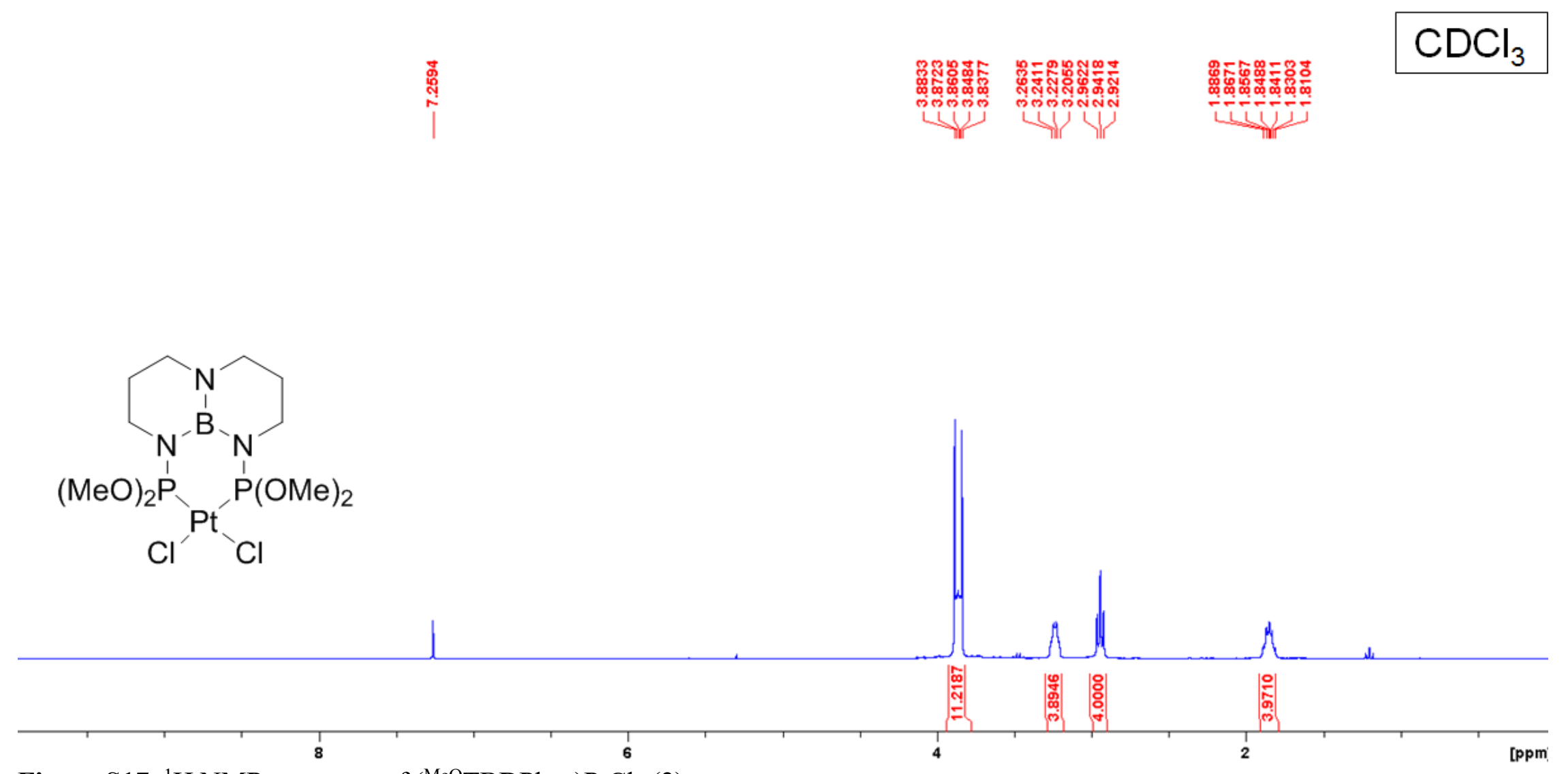

Figure S17. ${ }^{1} \mathrm{H}$ NMR spectrum of $\left({ }^{\mathrm{MeO}} \mathrm{TBDPhos}\right) \mathrm{PtCl}_{2}$ (2). 


\section{$\mathrm{CDCl}_{3}$}

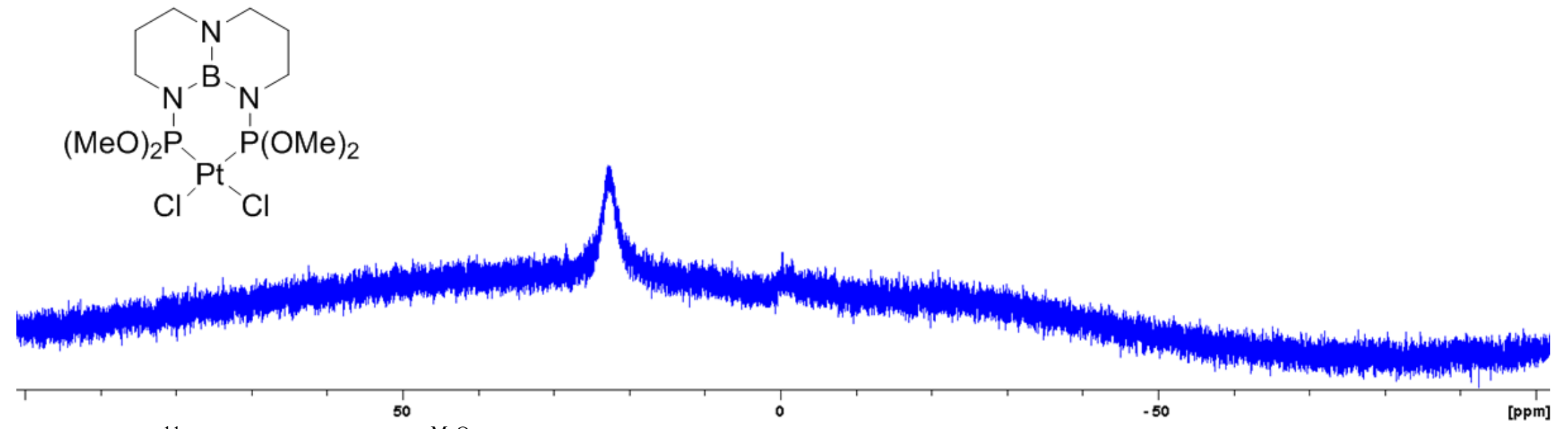

Figure S18. ${ }^{11} \mathrm{~B}$ NMR spectrum of $\left({ }^{\mathrm{MeO}} \mathrm{TBDPhos}\right) \mathrm{PtCl}_{2}$ (2). 


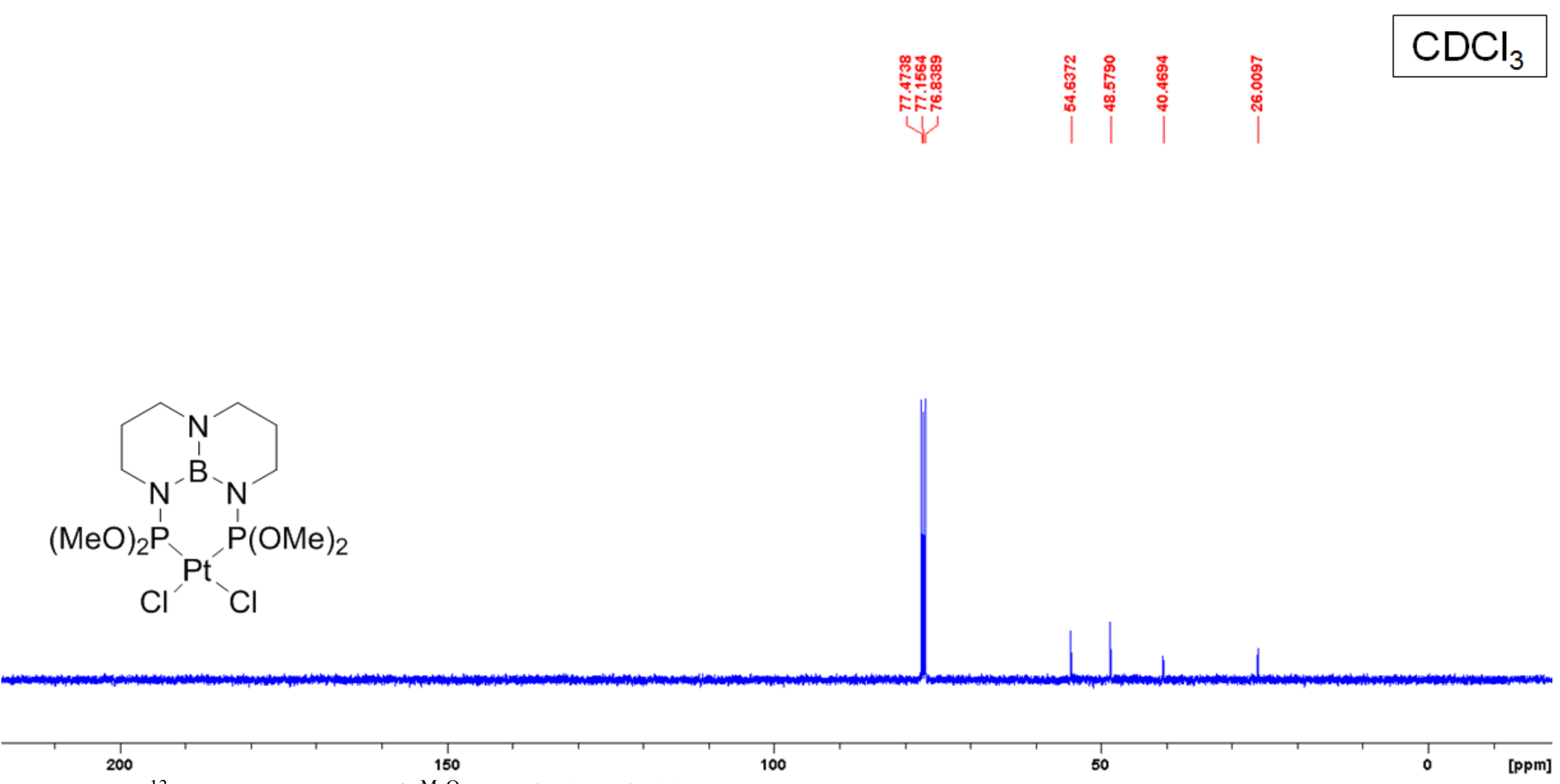

Figure S19. ${ }^{13} \mathrm{C}$ NMR spectrum of ( $\left.{ }^{\mathrm{MeO}} \mathrm{TBDPhos}\right) \mathrm{PtCl}_{2}$ (2). 


\section{$\mathrm{CDCl}_{3}$}

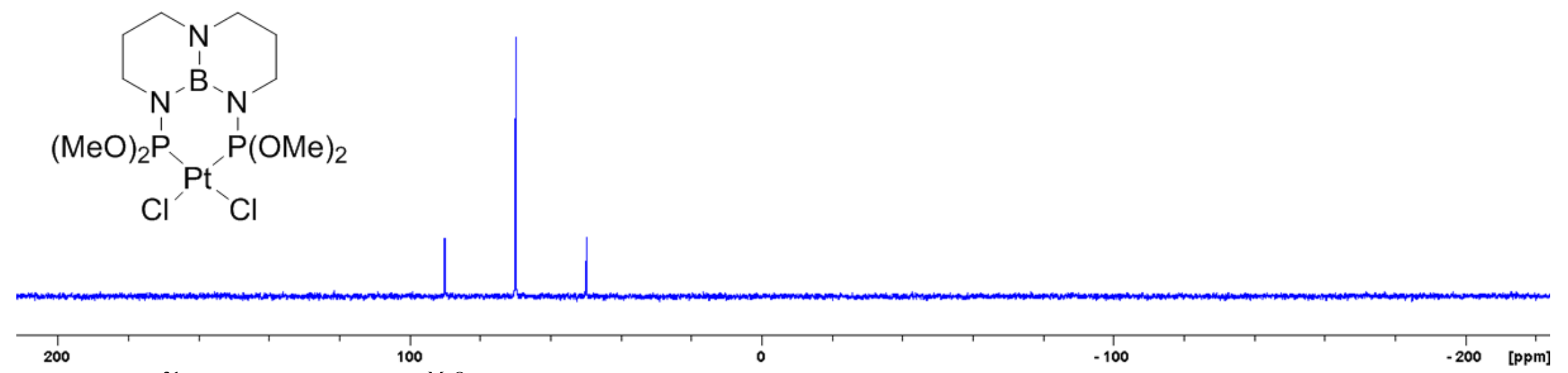

Figure S20. ${ }^{31} \mathrm{P}$ NMR spectrum of $\left({ }^{\mathrm{MeO}} \mathrm{TBDPhos}\right) \mathrm{PtCl}_{2}$ (2). 


\section{$\mathrm{CDCl}_{3}$}

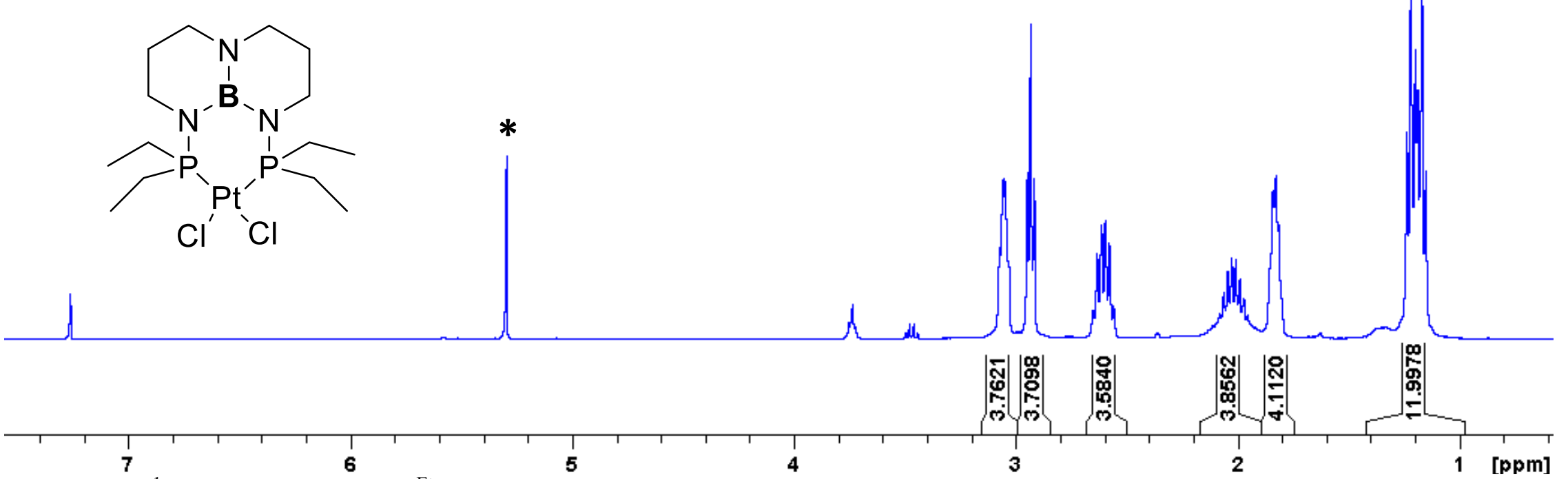

Figure S21. ${ }^{1} \mathrm{H}$ NMR spectrum of ( $\left.{ }^{\mathrm{Et}} \mathrm{TBDPhos}\right) \mathrm{PtCl}_{2}$ (3). The $*$ symbol is assigned to $\mathrm{CH}_{2} \mathrm{Cl}_{2}$, which likely co-crystallizes 3 as it does for 1 . 


\section{$\mathrm{CDCl}_{3}$}

욤

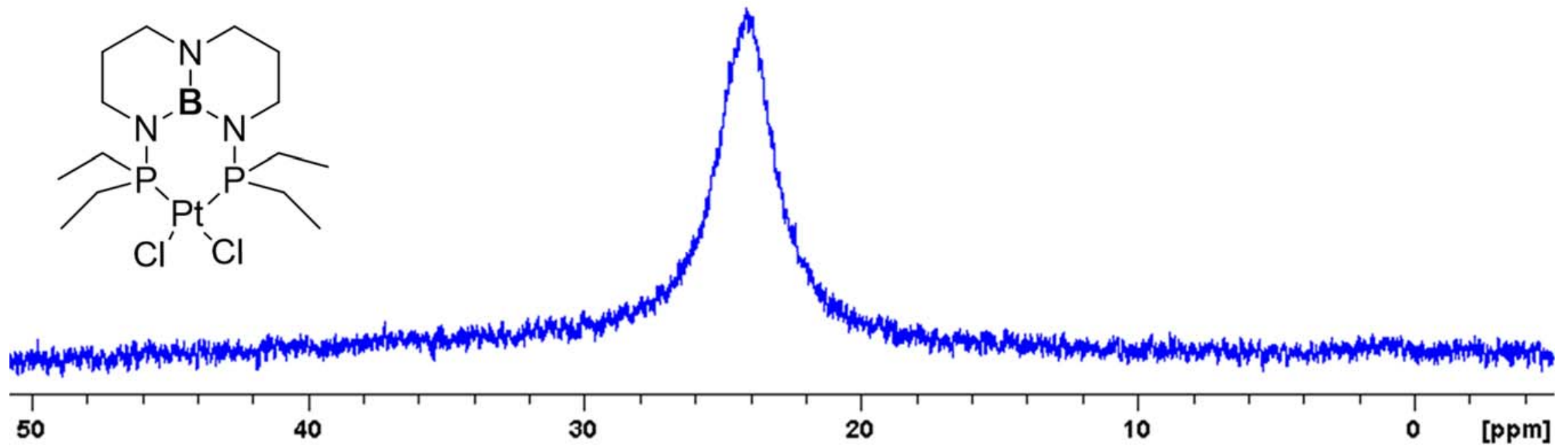

Figure S22. ${ }^{11} \mathrm{~B}$ NMR spectrum of ( $\left.{ }^{\mathrm{Et}} \mathrm{TBDPhos}\right) \mathrm{PtCl}_{2}$ (3). 
$\mathrm{CDCl}_{3}$

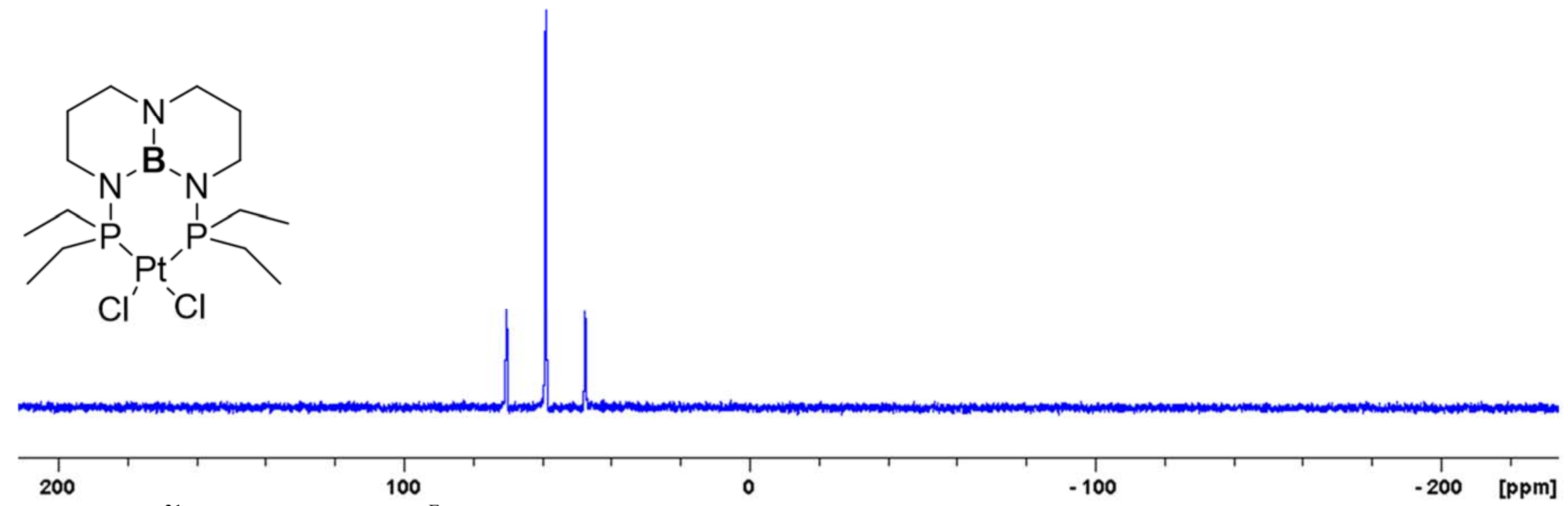

Figure S23. ${ }^{31} \mathrm{P}$ NMR spectrum of ( $\left.{ }^{\mathrm{Et}} \mathrm{TBDPhos}\right) \mathrm{PtCl}_{2}$ (3). 


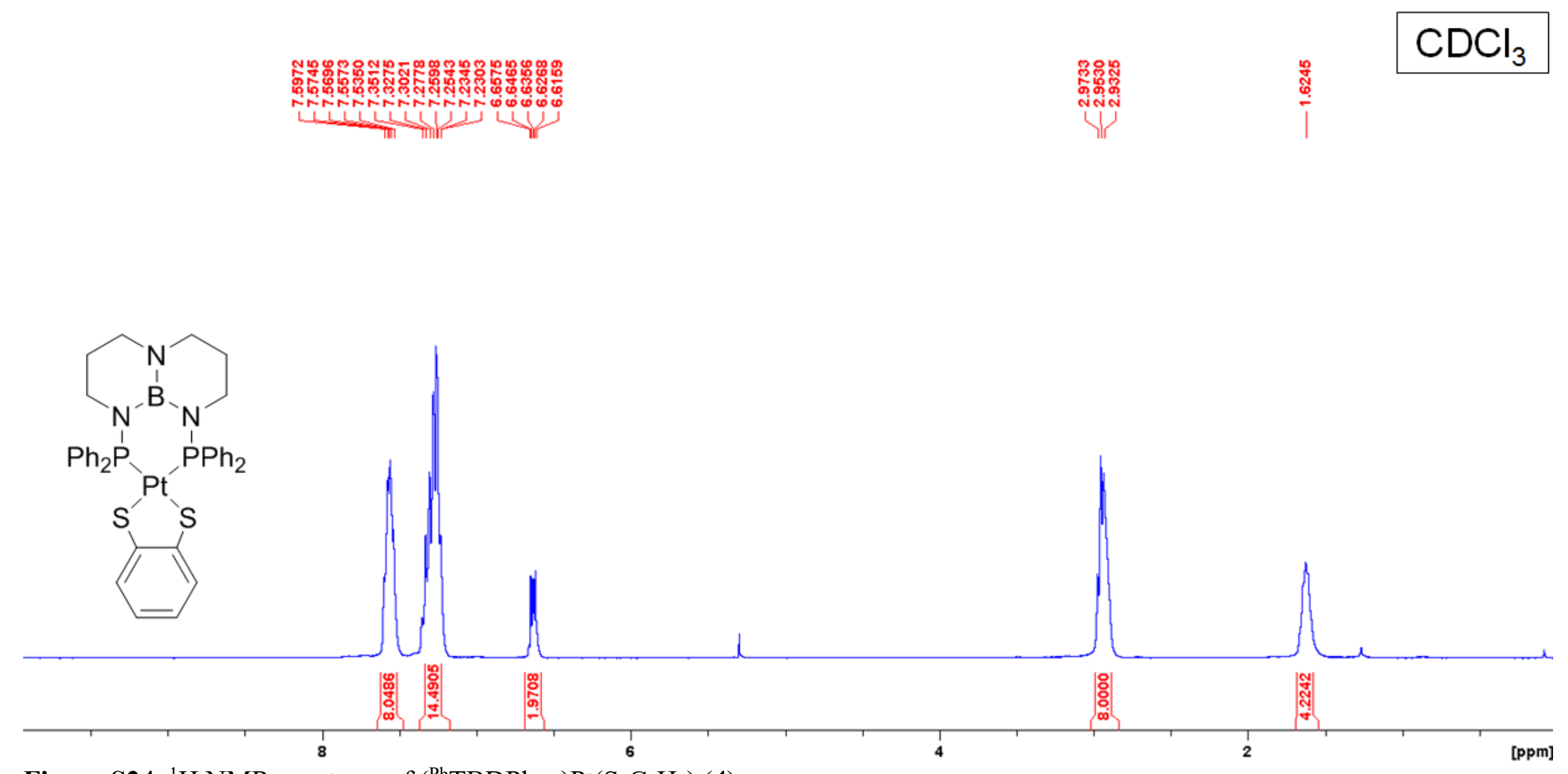

Figure S24. ${ }^{1} \mathrm{H}$ NMR spectrum of $\left({ }^{\mathrm{Ph}} \mathrm{TBDPhos}\right) \mathrm{Pt}\left(\mathrm{S}_{2} \mathrm{C}_{6} \mathrm{H}_{4}\right)$ (4). 


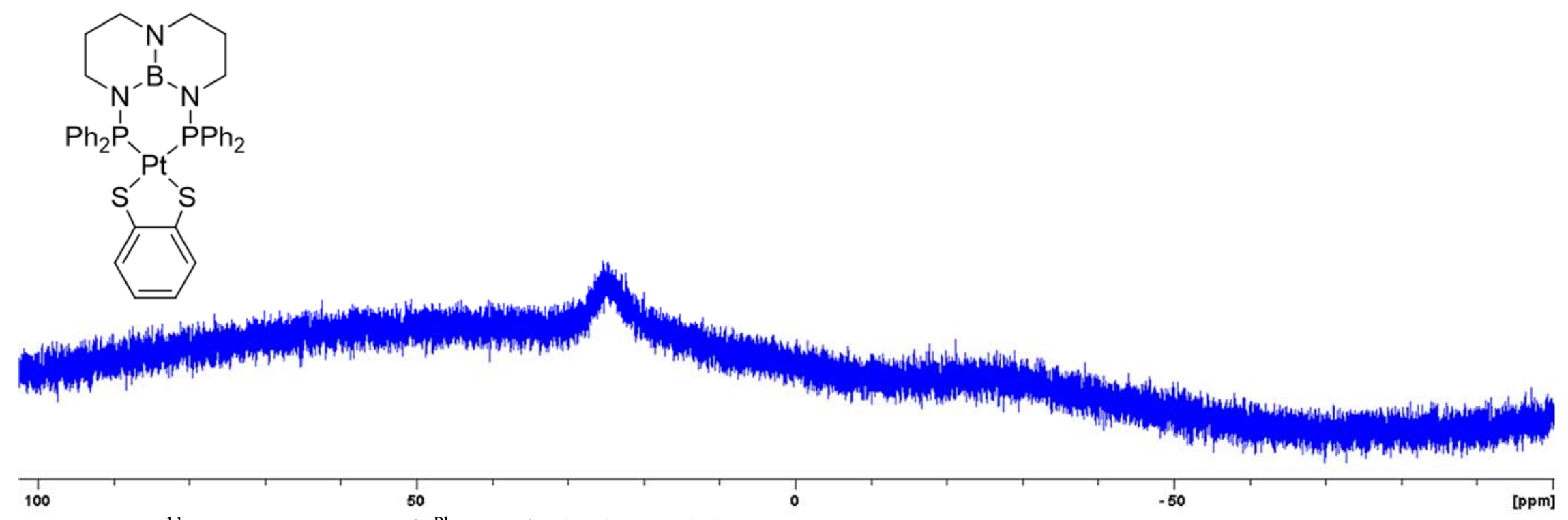

Figure S25. ${ }^{11} \mathrm{~B}$ NMR spectrum of $\left({ }^{\mathrm{Ph}} \mathrm{TBDPhos}\right) \mathrm{Pt}\left(\mathrm{S}_{2} \mathrm{C}_{6} \mathrm{H}_{4}\right)$ (4). 


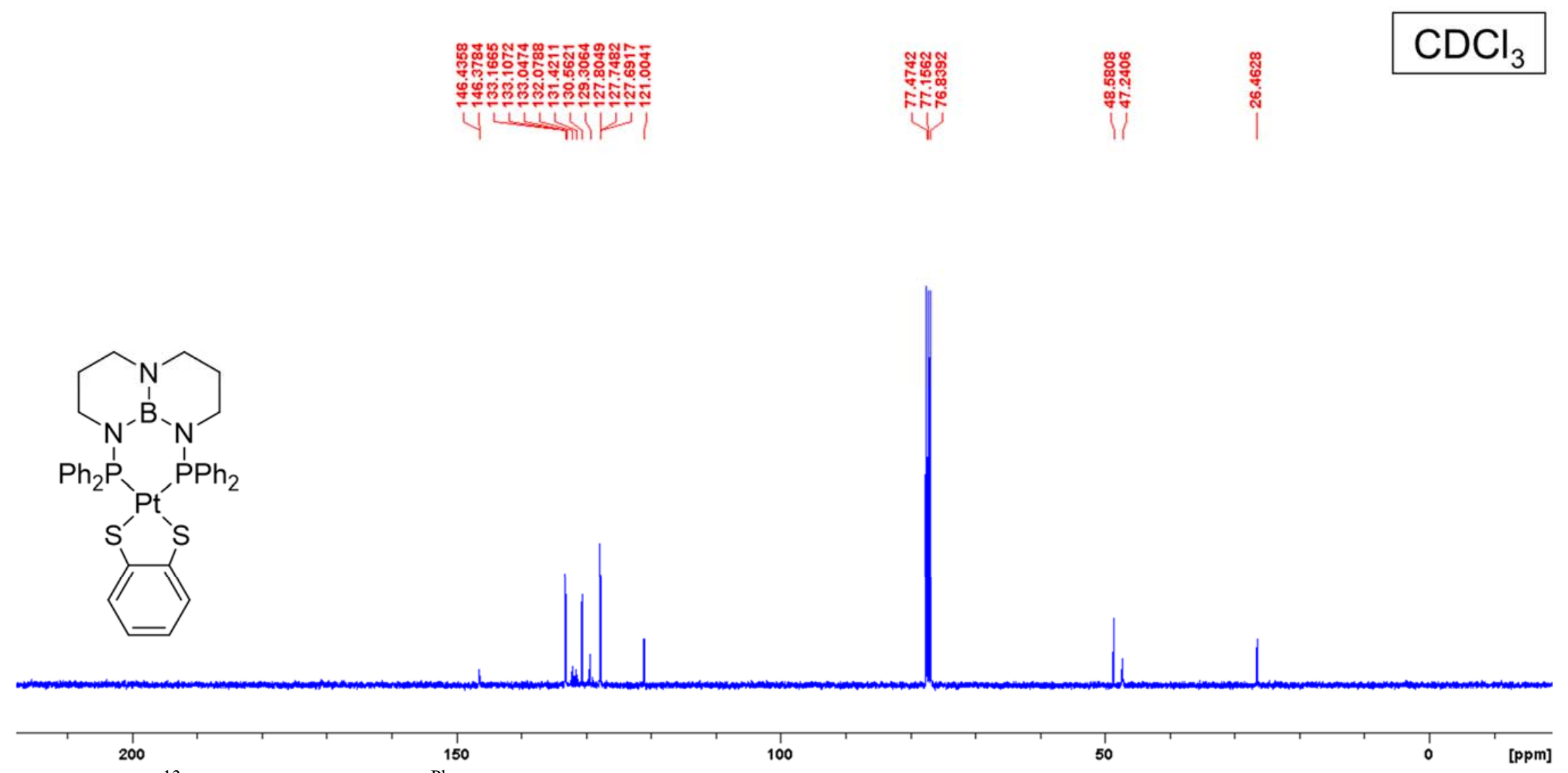

Figure S26. ${ }^{13} \mathrm{C}$ NMR spectrum of $\left({ }^{\mathrm{Ph}} \mathrm{TBDPhos}\right) \mathrm{Pt}\left(\mathrm{S}_{2} \mathrm{C}_{6} \mathrm{H}_{4}\right)$ (4). 


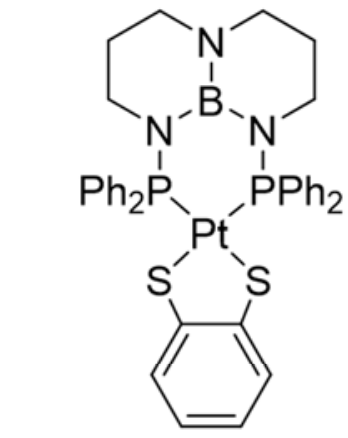

Figure S27. ${ }^{31} \mathrm{P}$ NMR spectrum of $\left({ }^{\mathrm{Ph}} \mathrm{TBDPhos}\right) \mathrm{Pt}\left(\mathrm{S}_{2} \mathrm{C}_{6} \mathrm{H}_{4}\right)$ (4). 


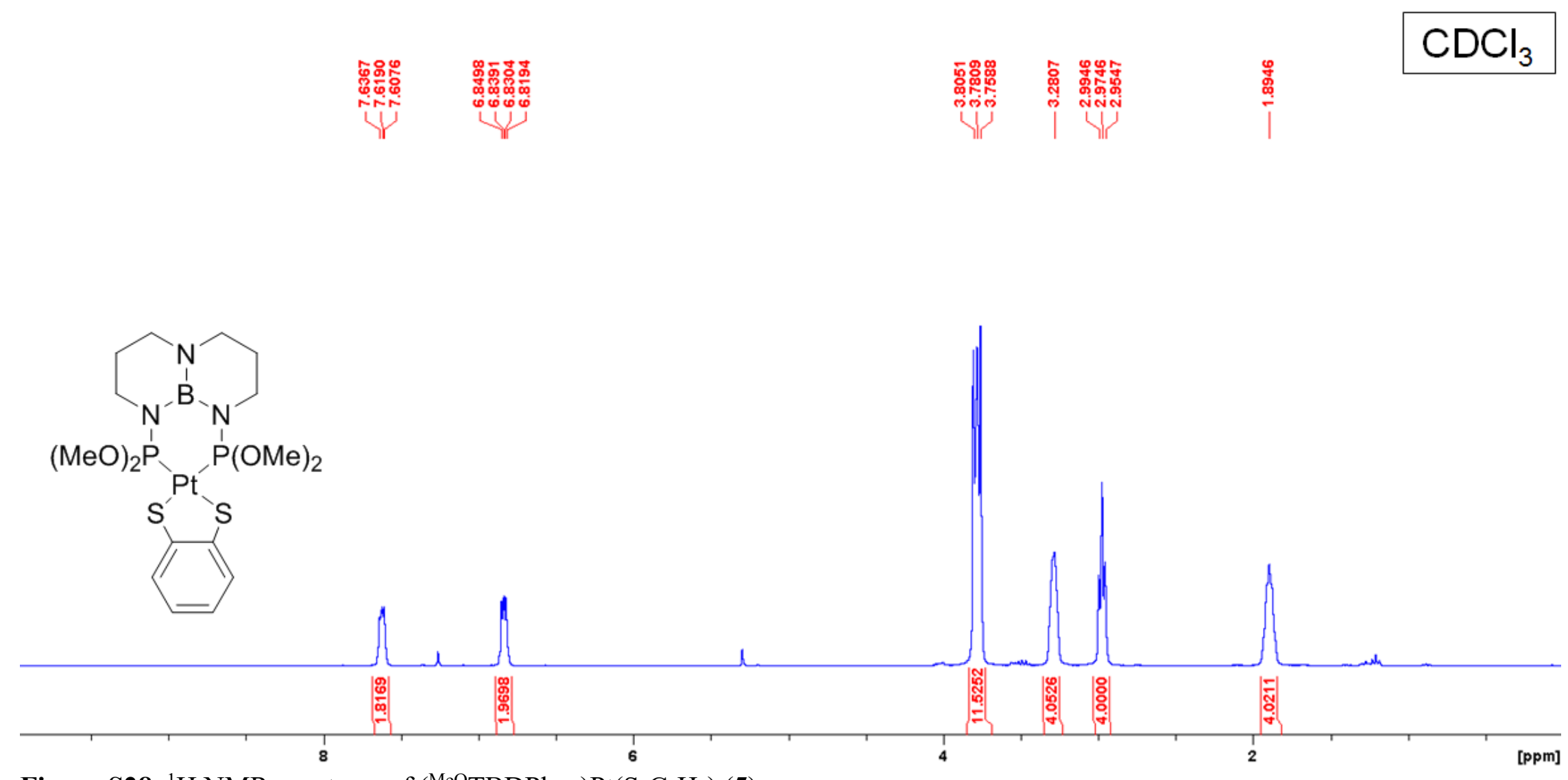

Figure S28. ${ }^{1} \mathrm{H}$ NMR spectrum of $\left({ }^{\mathrm{MeO}} \mathrm{TBDPhos}\right) \mathrm{Pt}\left(\mathrm{S}_{2} \mathrm{C}_{6} \mathrm{H}_{4}\right)(\mathbf{5})$. 

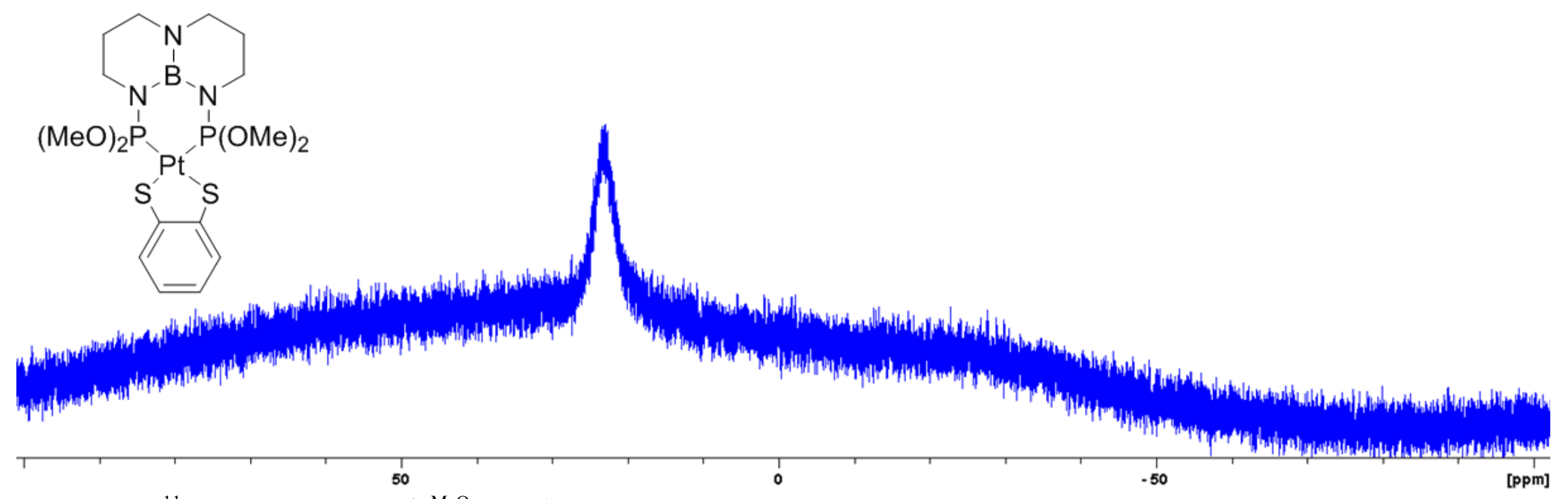

Figure S29. ${ }^{11} \mathrm{~B}$ NMR spectrum of $\left({ }^{\mathrm{MeO}} \mathrm{TBDPhos}\right) \mathrm{Pt}\left(\mathrm{S}_{2} \mathrm{C}_{6} \mathrm{H}_{4}\right)(\mathbf{5})$. 


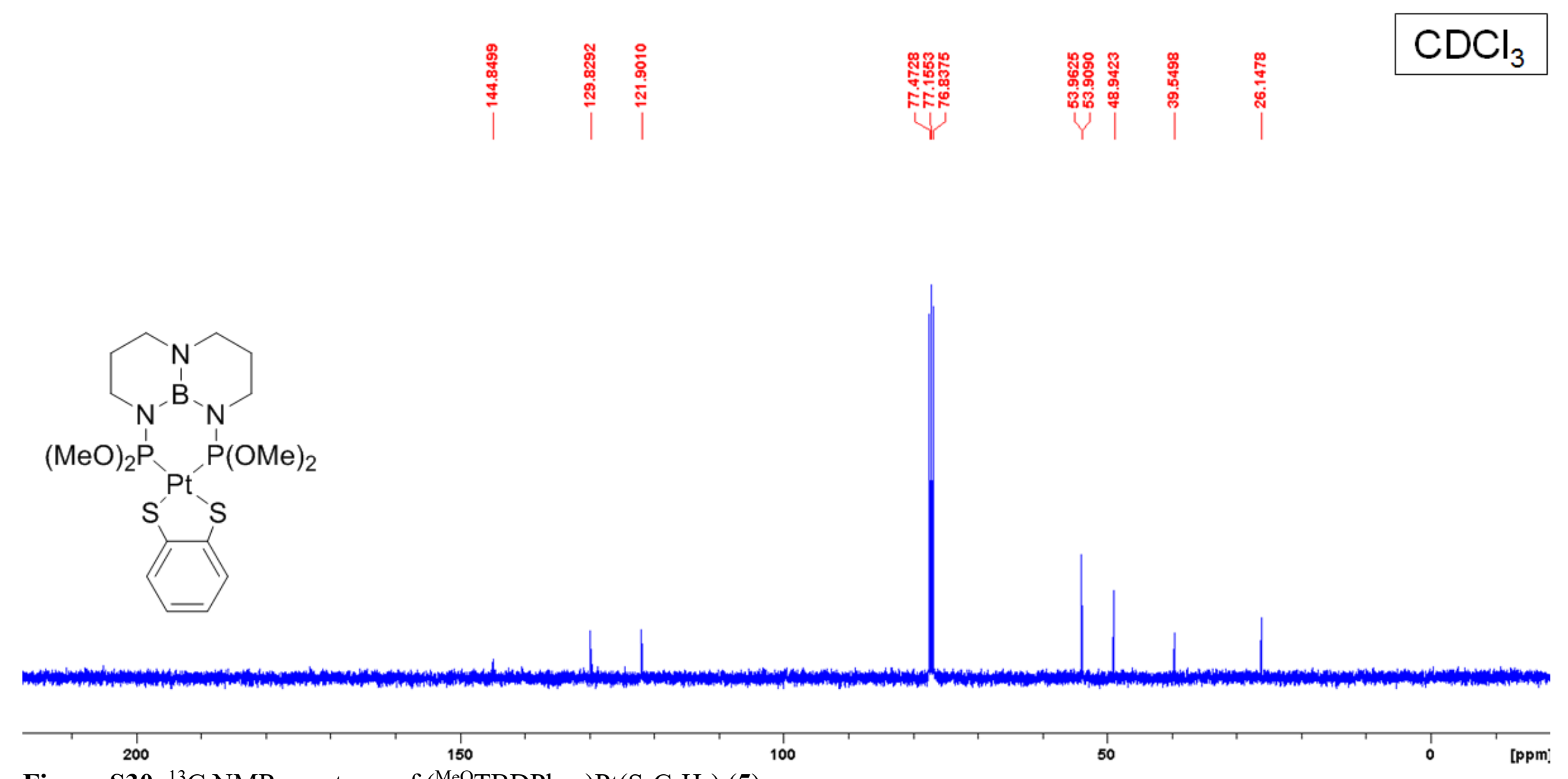

Figure S30. ${ }^{13} \mathrm{C}$ NMR spectrum of $\left({ }^{\mathrm{MeO}} \mathrm{TBDPhos}\right) \mathrm{Pt}\left(\mathrm{S}_{2} \mathrm{C}_{6} \mathrm{H}_{4}\right)(\mathbf{5})$. 


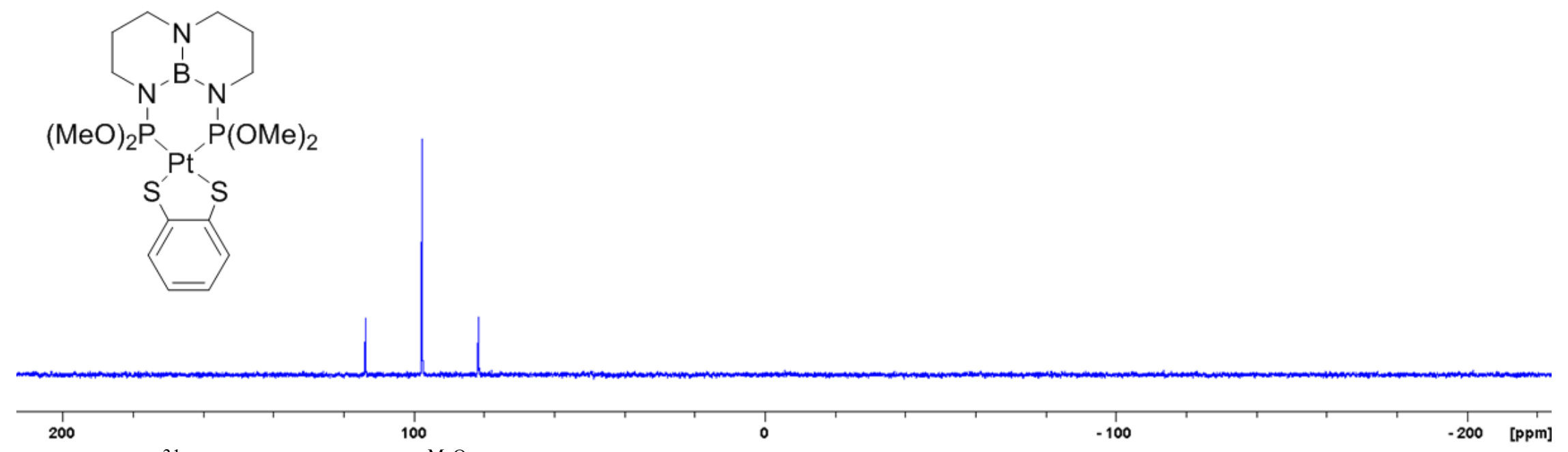

Figure S31. ${ }^{31} \mathrm{P}$ NMR spectrum of $\left({ }^{\mathrm{MeO}} \mathrm{TBDPhos}\right) \mathrm{Pt}\left(\mathrm{S}_{2} \mathrm{C}_{6} \mathrm{H}_{4}\right)$ (5). 


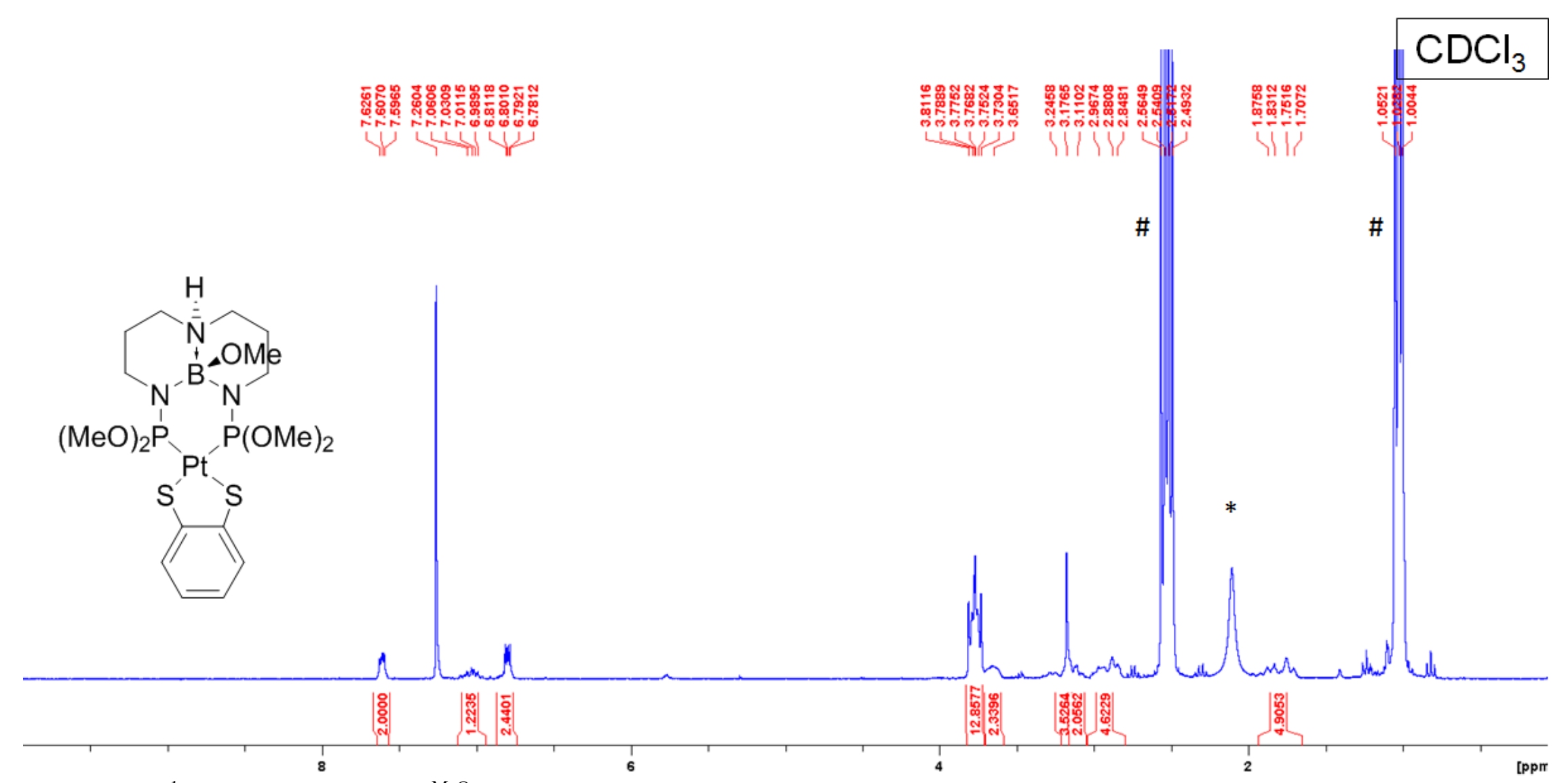

Figure S32. ${ }^{1} \mathrm{H}$ NMR spectrum of $\left({ }^{\mathrm{MeO}} \mathrm{TBDPhos}-\mathrm{MeOH}\right) \mathrm{Pt}\left(\mathrm{S}_{2} \mathrm{C}_{6} \mathrm{H}_{4}\right)(\mathbf{5 - M e O H})$. $\mathrm{NEt}_{3}$ was added to help dissolve the complex. The * and \# symbols indicate resonances assigned to residual $\mathrm{H}_{2} \mathrm{O}$ and added $\mathrm{NEt}_{3}$, respectively. 


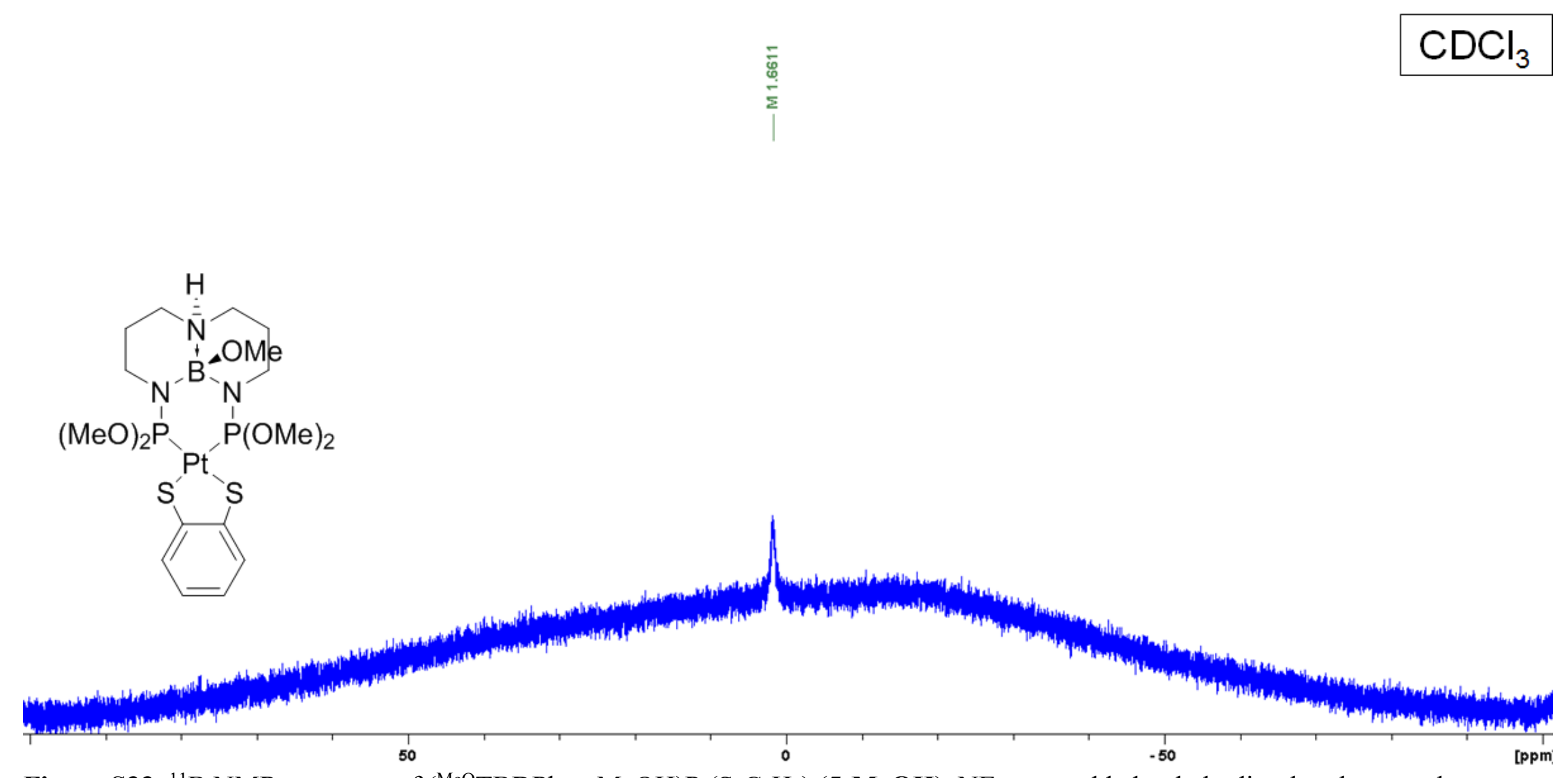

Figure S33. ${ }^{11} \mathrm{~B}$ NMR spectrum of $\left({ }^{\mathrm{MeO}} \mathrm{TBDPhos}-\mathrm{MeOH}\right) \mathrm{Pt}\left(\mathrm{S}_{2} \mathrm{C}_{6} \mathrm{H}_{4}\right)(\mathbf{5}-\mathbf{M e O H})$. $\mathrm{NEt}_{3}$ was added to help dissolve the complex. 


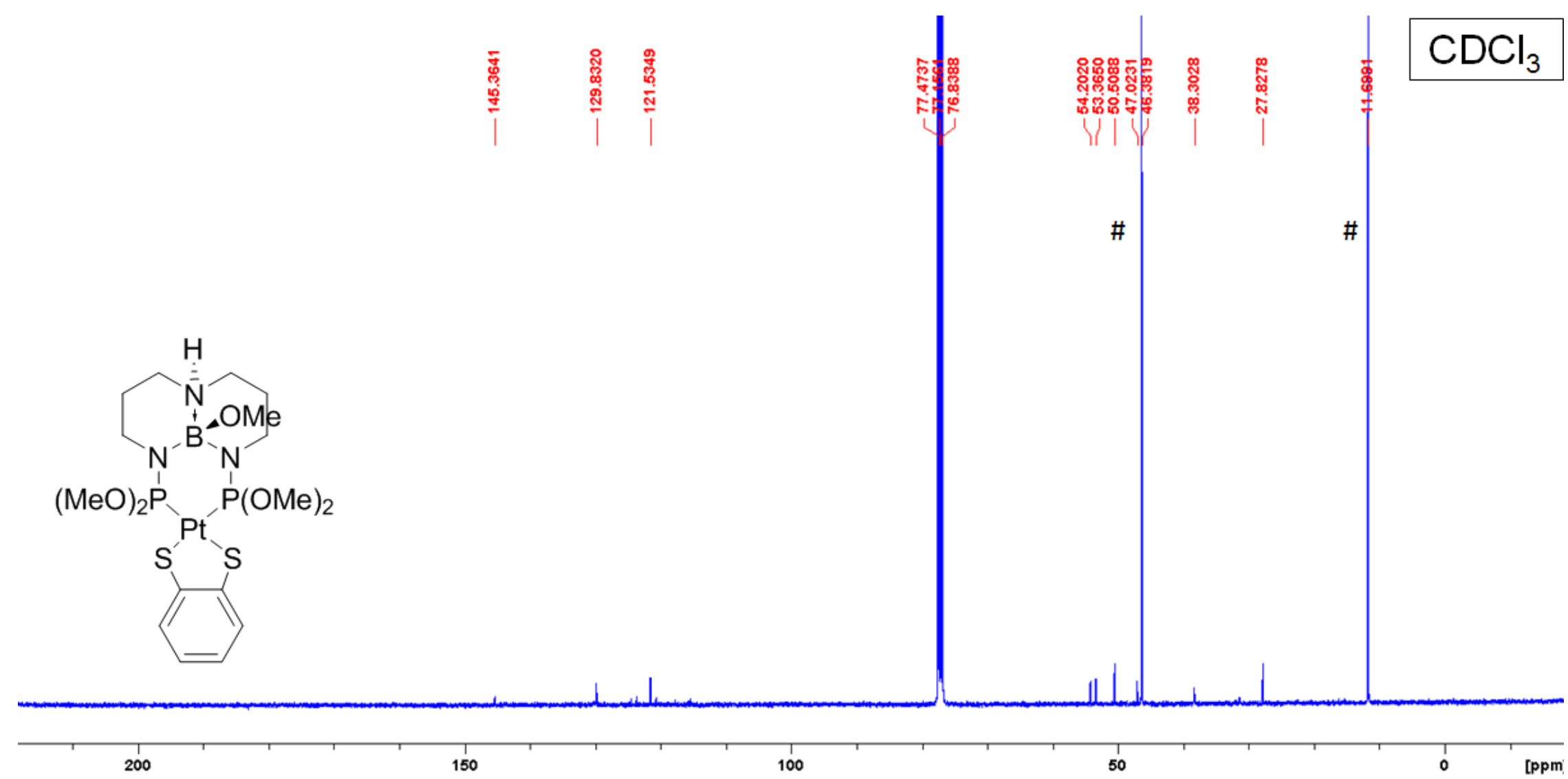

Figure S34. ${ }^{13} \mathrm{C}$ NMR spectrum of $\left({ }^{\mathrm{MeO}} \mathrm{TBDPhos}-\mathrm{MeOH}\right) \mathrm{Pt}\left(\mathrm{S}_{2} \mathrm{C}_{6} \mathrm{H}_{4}\right)($ 5-MeOH$)$. $\mathrm{NEt}_{3}$ was added to help dissolve the complex. The \# symbol indicates resonances assigned to added NEt3. 


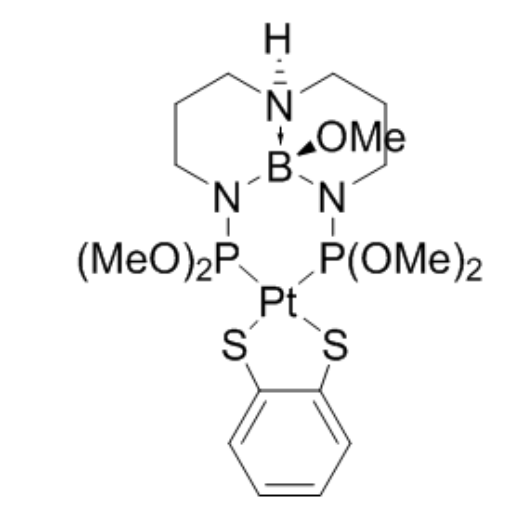

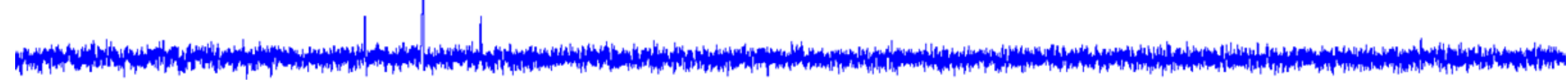
200 100

Figure S35. ${ }^{31} \mathrm{P}$ NMR spectrum of $\left({ }^{\mathrm{MeO}} \mathrm{TBDPhos}-\mathrm{MeOH}\right) \mathrm{Pt}\left(\mathrm{S}_{2} \mathrm{C}_{6} \mathrm{H}_{4}\right)(\mathbf{5 - M e O H})$. $\mathrm{NEt}_{3}$ was added to help dissolve the complex. 


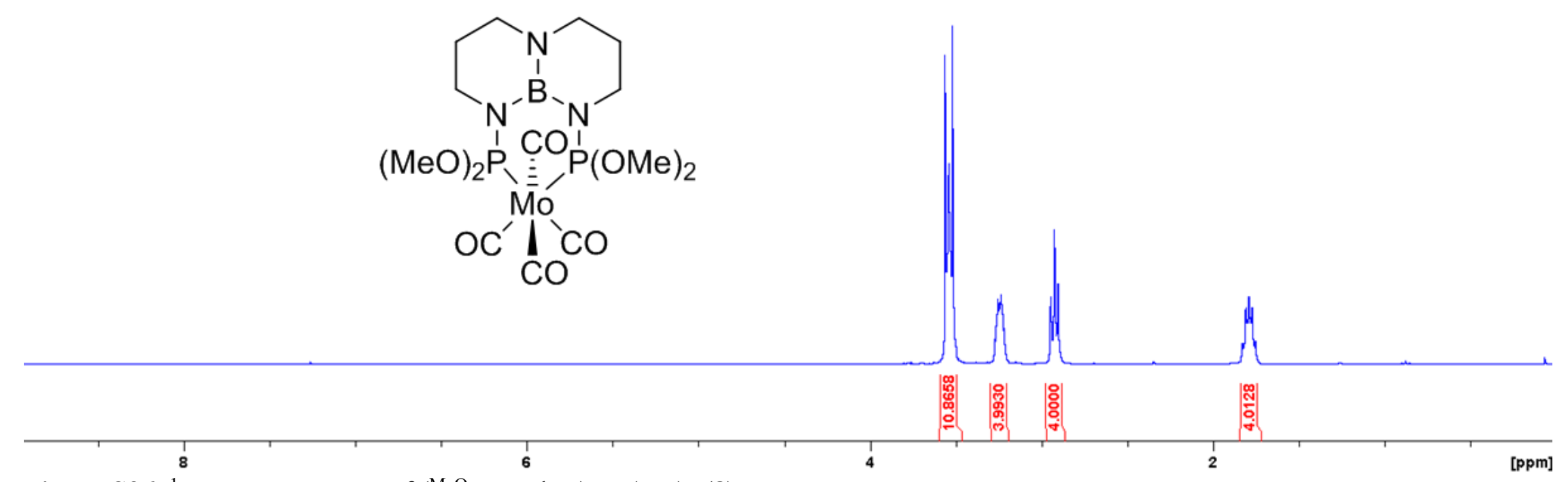

Figure S36. ${ }^{1} \mathrm{H}$ NMR spectrum of $\left({ }^{\mathrm{MeO}} \mathrm{TBDPhos}\right) \mathrm{Mo}(\mathrm{CO})_{4}(\mathbf{8})$. 


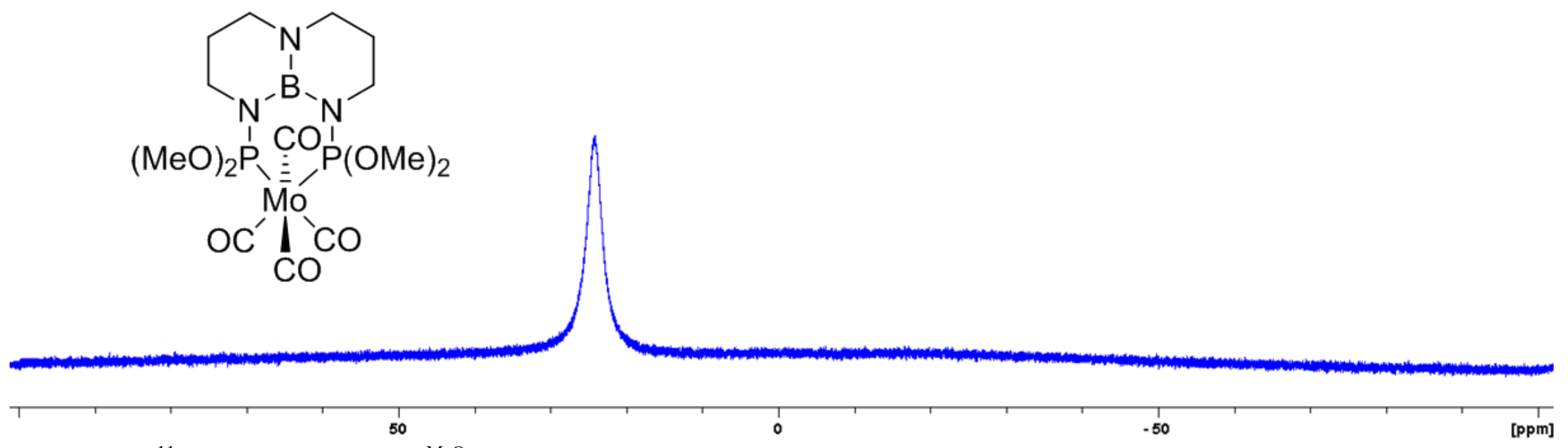

Figure S37. ${ }^{11} \mathrm{~B}$ NMR spectrum of $\left({ }^{\mathrm{MeO}} \mathrm{TBDPhos}\right) \mathrm{Mo}(\mathrm{CO})_{4}(\mathbf{8})$. 


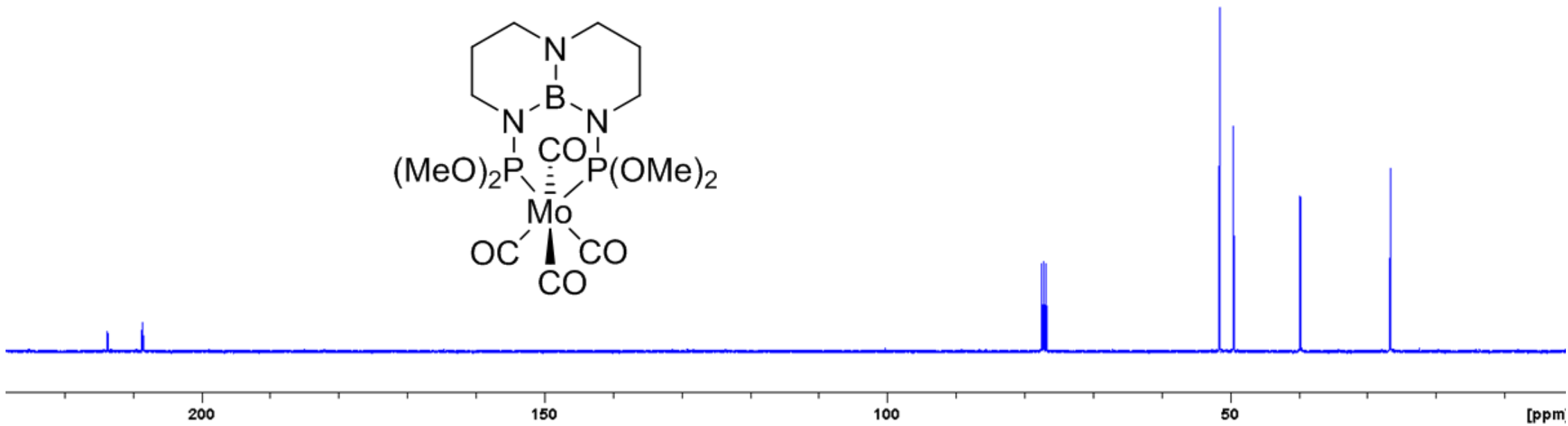

Figure S38. ${ }^{13} \mathrm{C}$ NMR spectrum of $\left({ }^{\mathrm{MeO}} \mathrm{TBDPhos}\right) \mathrm{Mo}(\mathrm{CO})_{4}(\mathbf{8})$. 


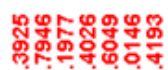

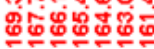

wilt

$\mathrm{CDCl}_{3}$

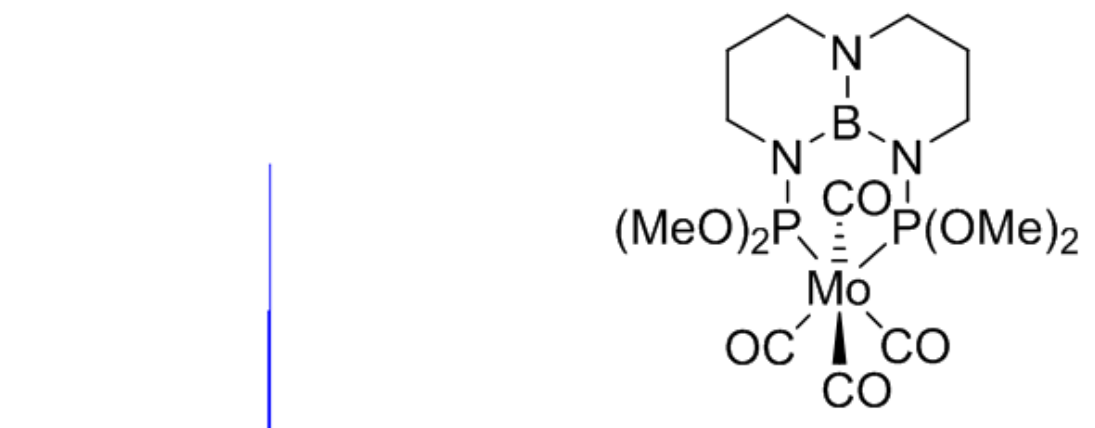

Figure S39. ${ }^{31} \mathrm{P}$ NMR spectrum of $\left({ }^{\mathrm{MeO}} \mathrm{TBDPhos}\right) \mathrm{Mo}(\mathrm{CO}) 4(\mathbf{8})$. 


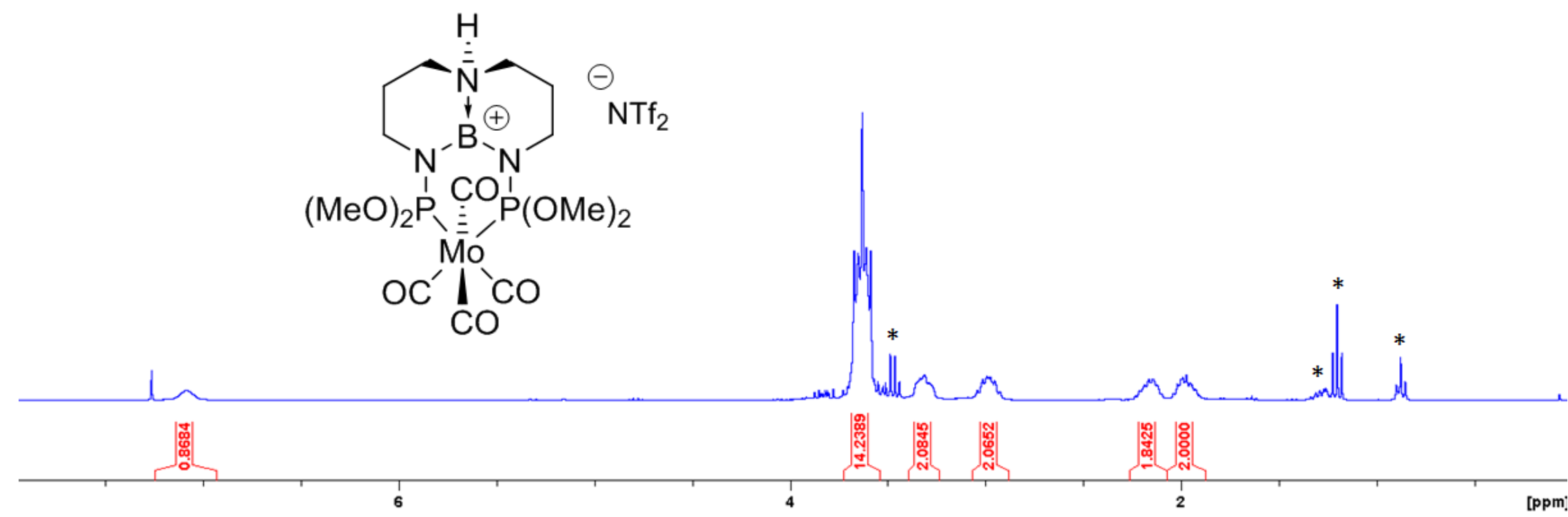

Figure S40. ${ }^{1} \mathrm{H}$ NMR spectrum of $\left({ }^{\mathrm{MeO}} \mathrm{TBDPhos}-\mathrm{HNTf} 2\right) \mathrm{Mo}(\mathrm{CO}) 4\left(\mathbf{8}-\mathbf{H N T f}_{2}\right)$. The ${ }^{*}$ symbol indicates resonances assigned to residual $\mathrm{Et}_{2} \mathrm{O}$ and pentane. 


\section{$\mathrm{CDCl}_{3}$}
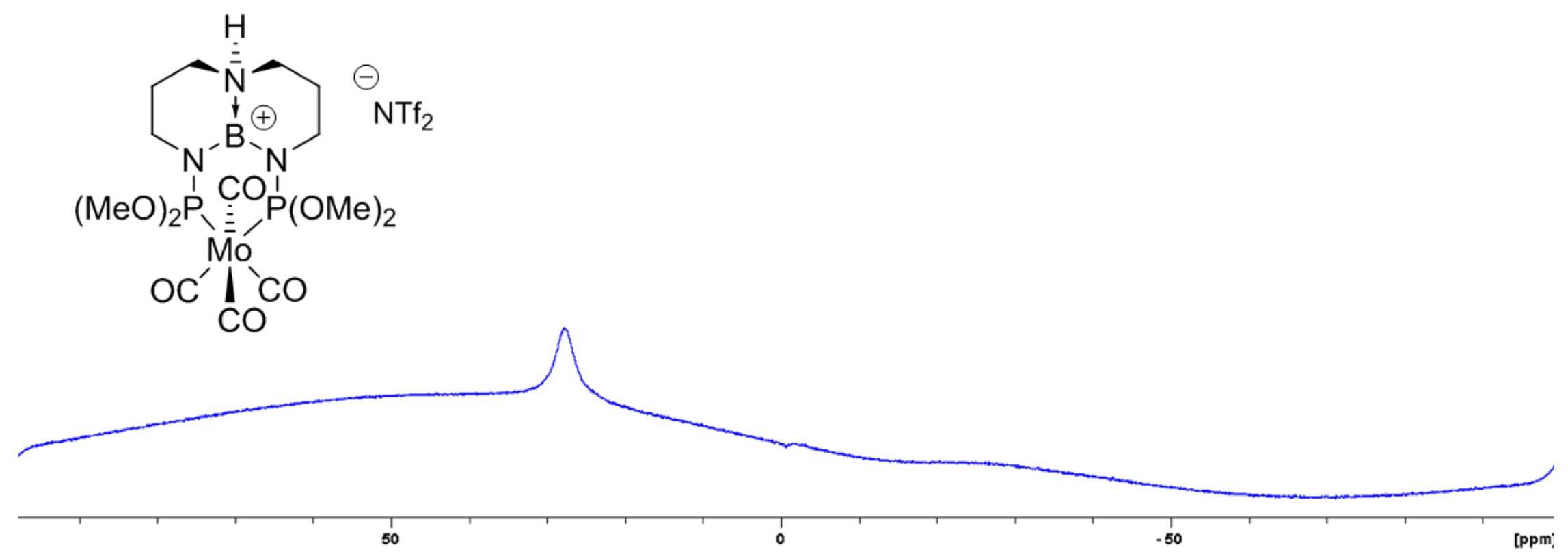

Figure S41. ${ }^{11} \mathrm{~B}$ NMR spectrum of $\left({ }^{\mathrm{MeO}} \mathrm{TBDPhos}_{-\mathrm{HNTf}}\right) \mathrm{Mo}(\mathrm{CO}) 4\left(\mathbf{8}-\mathrm{HNTf}_{2}\right)$. 


\section{$\mathrm{CDCl}_{3}$}

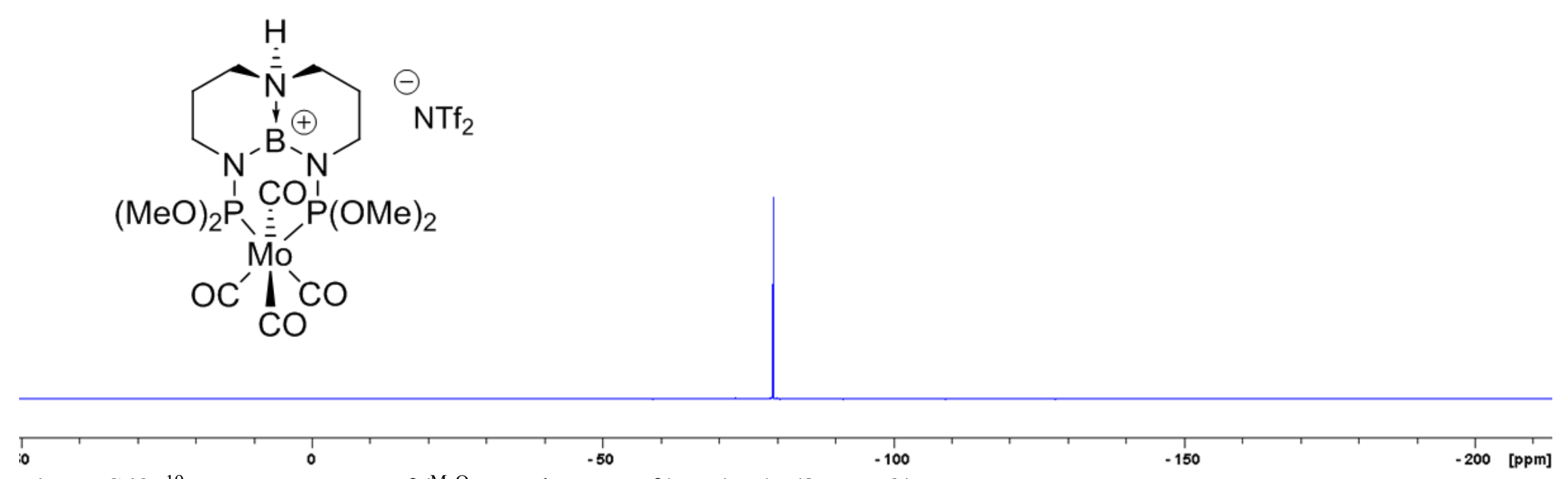

Figure S42. ${ }^{19} \mathrm{~F}$ NMR spectrum of $\left({ }^{\mathrm{MeO}} \mathrm{TBDPhos}-\mathrm{HNTf}_{2}\right) \mathrm{Mo}(\mathrm{CO}) 4\left(\mathbf{8}-\mathrm{HNTf}_{2}\right)$. 


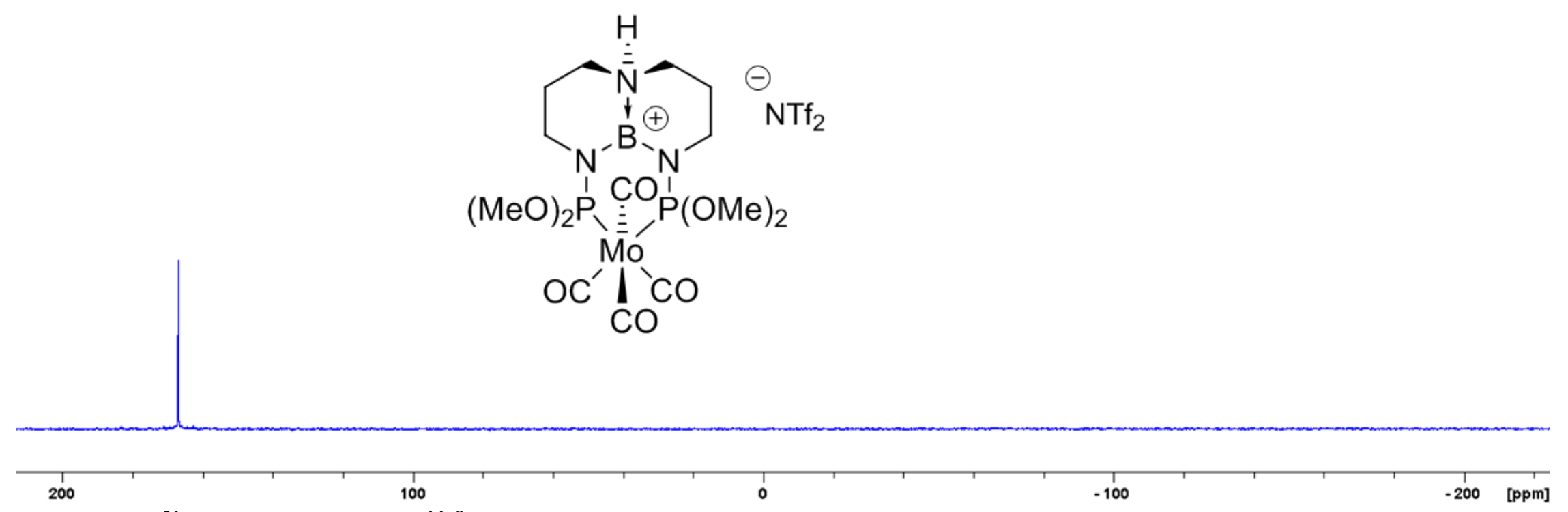

Figure S43. ${ }^{31} \mathrm{P}$ NMR spectrum of $\left({ }^{\mathrm{MeO}} \mathrm{TBDPhos}^{-H N T f_{2}}\right) \mathrm{Mo}(\mathrm{CO}) 4\left(\mathbf{8}-\mathrm{HNTf}_{2}\right)$. 


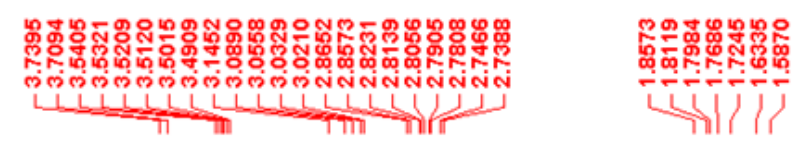

$\mathrm{CDCl}_{3}$

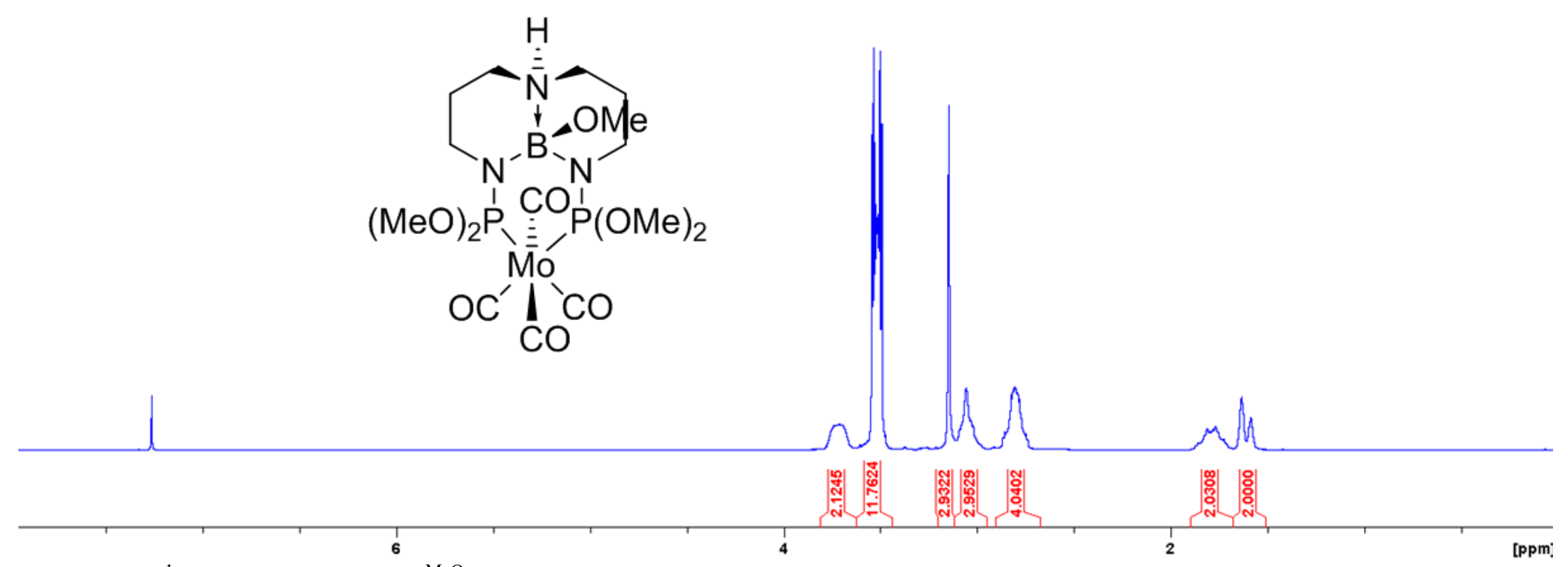

Figure S44. ${ }^{1} \mathrm{H}$ NMR spectrum of $\left({ }^{\mathrm{MeO}} \mathrm{TBDPhos}-\mathrm{MeOH}\right) \mathrm{Mo}(\mathrm{CO}) 4(\mathbf{8}-\mathbf{M e O H})$. 


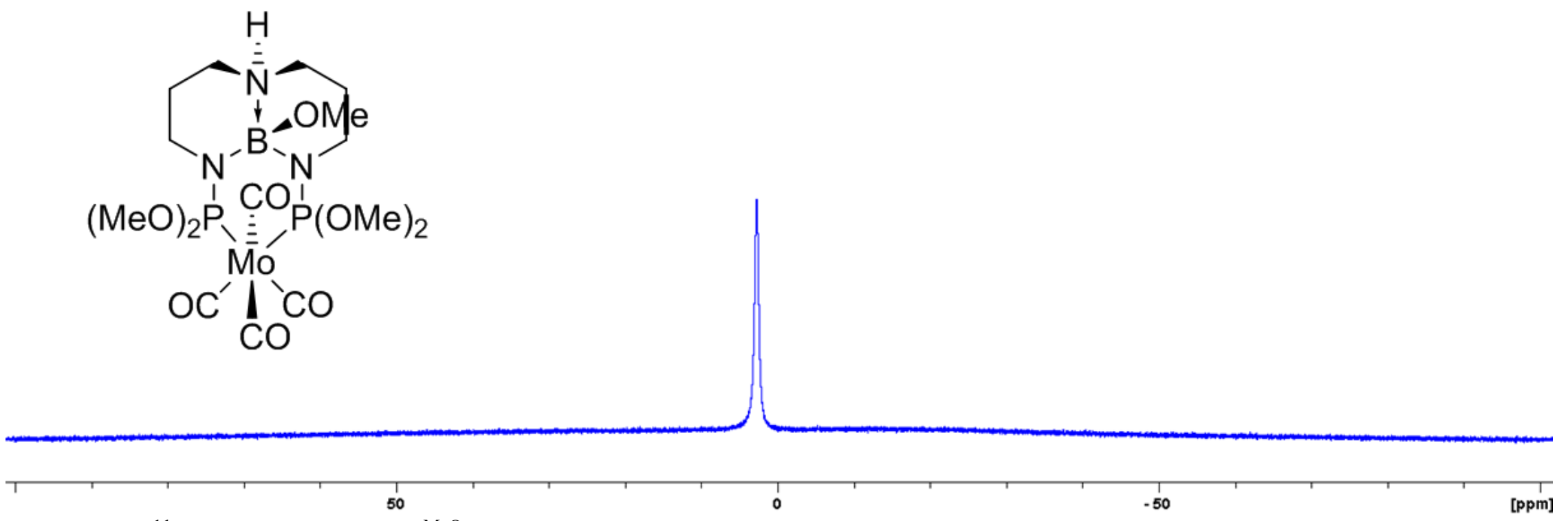

Figure S45. ${ }^{11} \mathrm{~B}$ NMR spectrum of $\left({ }^{\mathrm{MeO}} \mathrm{TBDPhos}-\mathrm{MeOH}\right) \mathrm{Mo}(\mathrm{CO}) 4(\mathbf{8}-\mathbf{M e O H})$. 


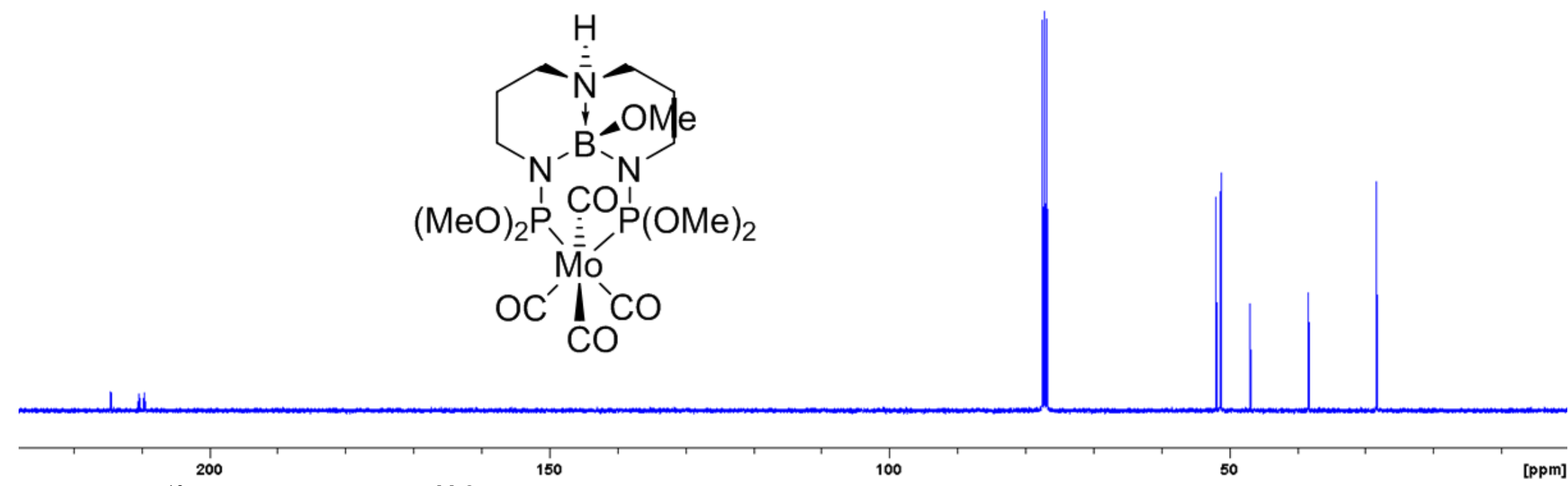

Figure S46. ${ }^{13} \mathrm{C}$ NMR spectrum of $\left({ }^{\mathrm{MeO}} \mathrm{TBDPhos}-\mathrm{MeOH}\right) \mathrm{Mo}(\mathrm{CO})_{4}(\mathbf{8}-\mathbf{M e O H})$. 


\section{$\mathrm{CDCl}_{3}$}

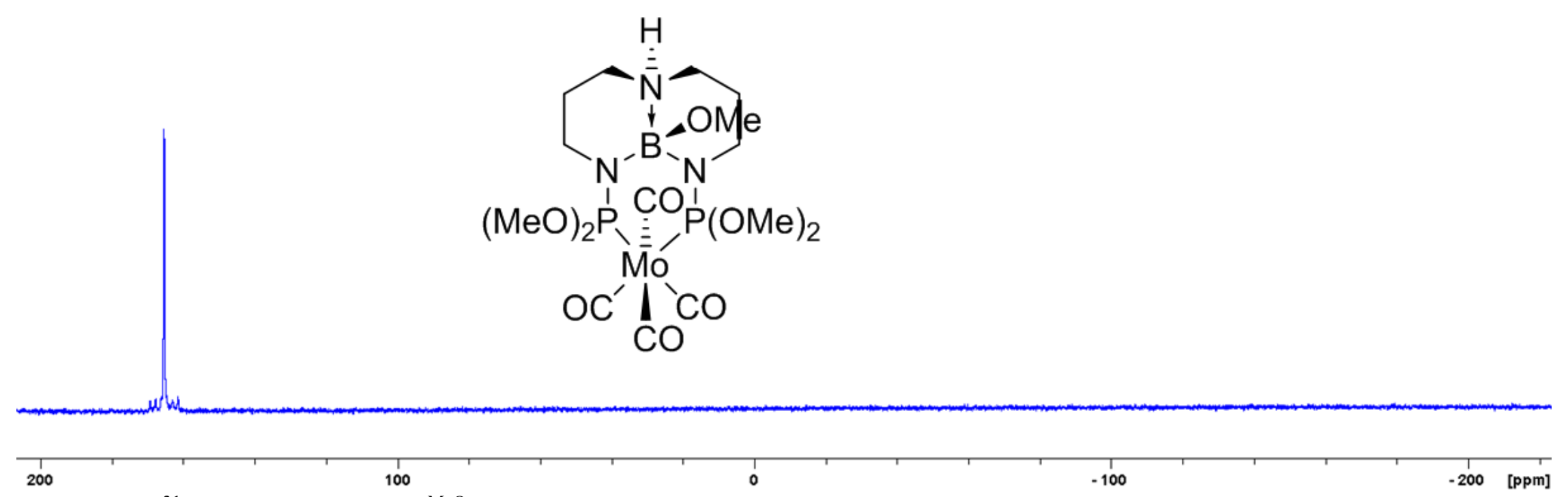

Figure S47. ${ }^{31} \mathrm{P}$ NMR spectrum of $\left({ }^{\mathrm{MeO}} \mathrm{TBDPhos}-\mathrm{MeOH}\right) \mathrm{Mo}(\mathrm{CO}) 4(\mathbf{8}-\mathbf{M e O H})$. 


\section{Supporting Information References}

(1) Lee, K.; Donahue, C. M.; Daly, S. R. Triaminoborane-bridged diphosphine complexes with Ni and Pd: coordination chemistry, structures, and ligand-centered reactivity. Dalton Trans. 2017, 46, 9394-9406.

(2) Mazour, Z. Substituted phosphorochloridous acid. Ger. Offen. DE 2643442, April 7, 1977.

(3) Lee, K.; Wei, H.; Blake, A. V.; Donahue, C. M.; Keith, J. M.; Daly, S. R. Ligand K-edge XAS, DFT, and TDDFT analysis of pincer linker variations in Rh(I) PNP complexes: reactivity insights from electronic structure. Dalton Trans. 2016, 45, 97749785.

(4) Sheldrick, G. M. SHELXT - Integrated space-group and crystal-structure determination. Acta Crystallogr., Sect. A Found. Adv. 2015, 71, 3-8.

(5) Sheldrick, G. M. Crystal structure refinement with SHELXL. Acta Crystallogr., Sect. C Struct. Chem. 2015, 71, 3-8.

(6) (a) Dolomanov, O. V.; Bourhis, L. J.; Gildea, R. J.; Howard, J. A. K.; Puschmann, H. OLEX2: a complete structure solution, refinement and analysis program. J. Appl. Crystallogr. 2009, 42, 339-341. (b) Macrae, C. F.; Bruno, I. J.; Chisholm, J. A.; Edgington, P. R.; McCabe, P.; Pidcock, E.; Rodriguez-Monge, L.; Taylor, R.; van de Streek, J.; Wood, P. A. Mercury CSD 2.0 new features for the visualization and investigation of crystal structures. J. Appl. Crystallogr. 2008, 41, 466-470.

(7) (a) Weigend, F.; Ahlrichs, R. Balanced basis sets of split valence, triple zeta valence and quadruple zeta valence quality for H to Rn: Design and assessment of accuracy. Phys. Chem. Chem. Phys. 2005, 7, 3297-3305. (b) Zhao, Y.; Truhlar, D. G. A new local density functional for main-group thermochemistry, transition metal bonding, thermochemical kinetics, and noncovalent interactions. J. Chem. Phys. 2006, 125, 194101/194101-194101/194118.

(8) Becke, A. D. A new mixing of Hartree-Fock and local-density-functional theories. J. Chem. Phys. 1993, 98, $1372-1377$.

(9) Grimme, S.; Antony, J.; Ehrlich, S.; Krieg, H. A consistent and accurate ab initio parametrization of density functional dispersion correction (DFT-D) for the 94 elements H-Pu. J. Chem. Phys. 2010, 132, 154104/154101-154104/154119. 